
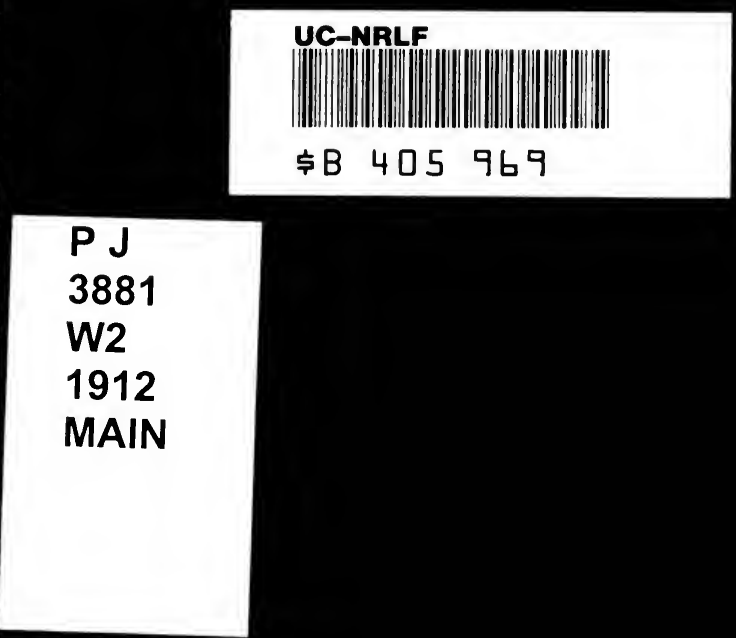


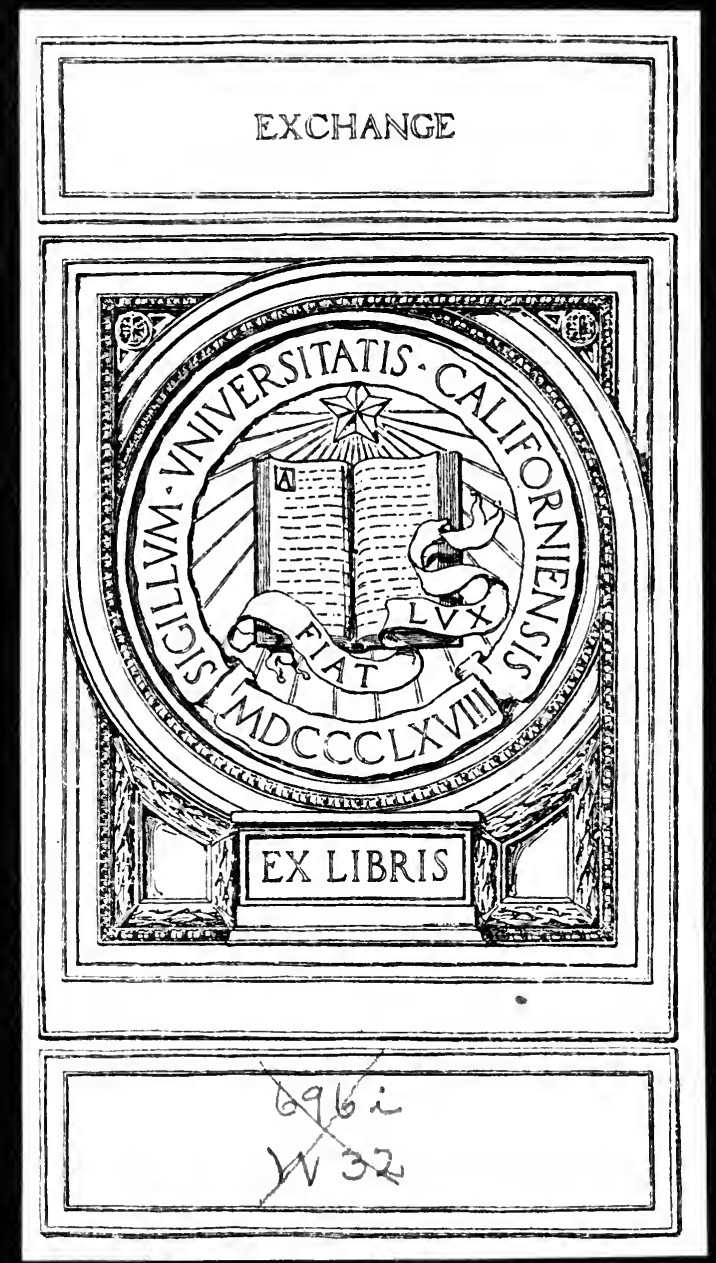




\section{SOME KOUYUNJIK LETTERS AND RELATED TEXTS}

\section{A DISSERTATION}

SUBMITTED TO THE FACULTY OF THE GRADUATE DIVINITY SCHOOL IN CANDIDACY FOR THE DEGREE OF DOCTOR OF PHILOSOPHY

(DEPARTMENT OF SEMITIC LANGUAGES AND LITERATURES)

BY

\section{LEROY WATERMAN}

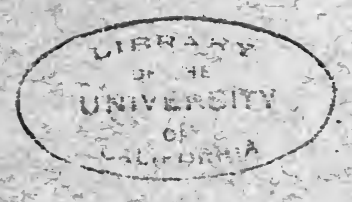

Reprinted from

The American Journal of Semitic Languages and Literatures Vol. XXVIII, No. 2, and Vol. XXIX, No. r, rgi2 

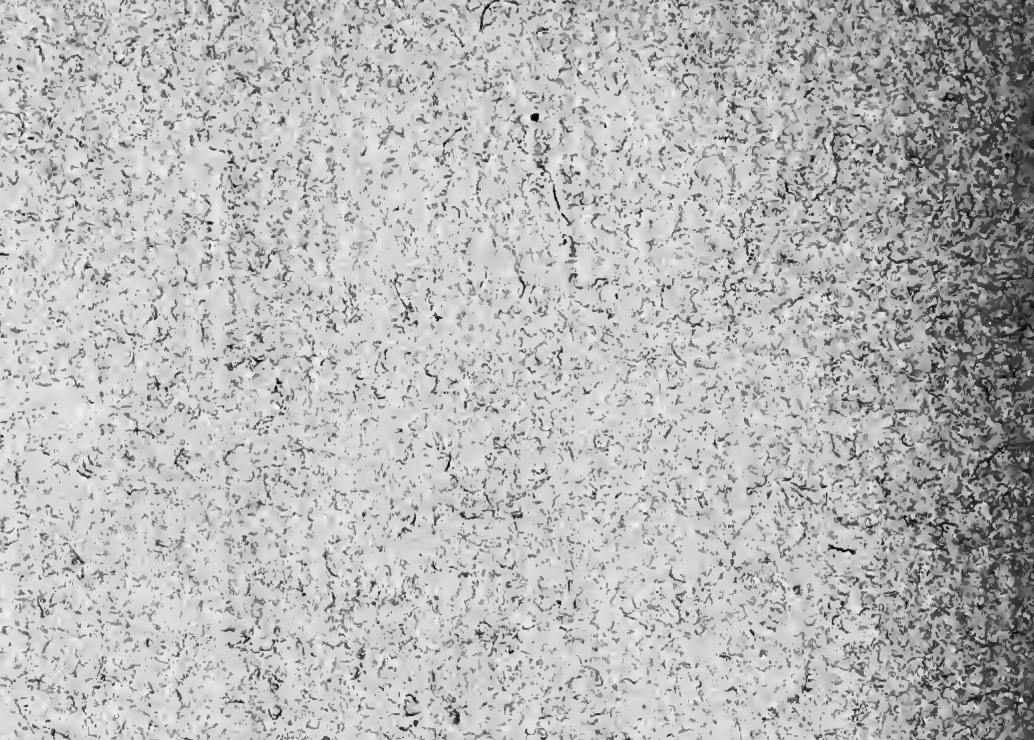

station

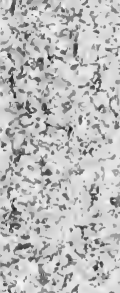

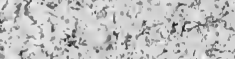

$4 \rightarrow t^{4}$

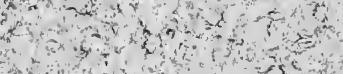




\section{Tbe University of Cbicago}

FOUNDED QY JOHN D. ROCKEFELLER

\section{SOME KOUYUNJIK LETTERS AND RELATED TEXTS}

\section{A DISSERTATION}

SUBMITTED TO THE FACULTY OF THE GRADUATE DIVINITY

SCHOOL IN CANDIDACY FOR THE DEGREE OF DOCTOR OF PHILOSOPHY

(DEPARTMENT OF SEMITIC LANGUAGES AND LITERATURES)

BY

\section{LEROY WATERMAN}

\section{Reprinted from}

The American Journal of Semitic Languages and Literatures Vol. XXVIII, No. 2, and Vol. XXIX, No. 1, I9I 2 


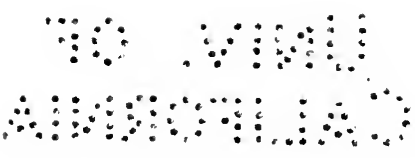




\section{VITA}

I, Leroy Waterman, was born at Pierpont, Ohio, July 4, 1875. After completing my preparation for college in New Lyme Institute at South New Lyme, Ohio, in 1894, I attended Hillsdale College, at Hillsdale, Michigan and received the degree of A.B. in 1898 and the degree of B.D. in 1900 from the Theological School at Hillsdale. The two following years were spent on Old Testament studies in the University of Oxford. Since that time I have been Professor of Old Testament Language and Literature in Hillsdale College. The year 1906-7 was spent in study, on leave of absence, in the University of Berlin, during which especial attention was given to Semitics and Comparative Religion. During the summer quarters of 1909 and 1910 I was in residence at the University of Chicago. In the Autumn of 1910 I was appointed Fellow in Semitics and spent from January to September 1911 in the study of cuneiform tablets in the British Museum. I was reappointed Fellow in October, 1911 and again in June, 1912. While a student in The University of Chicago I attended the courses of Professors Breasted, Foster, Price, and J. M. P. Smith.

In acknowledging my gratitude to all my teachers, I wish to make special mention of Professors Friederich Delitzsch, S. R. Driver, G. B. Gray and Otto Pfleiderer. To all of these my thanks are due, but pre-eminently to Professor Robert Francis Harper, under whose direction I have been permitted to complete my graduate work, do I owe more than I can well express. However, for the results of this paper and the views therein expressed, I alone am responsible. 
Digitized by the Internet Archive in 2007 with funding from Microsoft Corporation 


\section{THE AMERICAN JOURNAL}

\section{OF \\ SEMITIC LANGUAGES AND LITERATURES}

(CONTINUING HEBRAICA)

Volume XXIX OCTOBER, 1912 Number 1

\section{SOME KOUYUNJIK LETTERS AND RELATED TEXTS}

By Leroy Waterman

Among others, the following texts of the Kouyunjik collections in the British Museum were copied by me during a stay in London in 1911. According to the catalogue these texts with three exceptions may be classified as letters. The exceptions are K. 1545 and 83-1-18, 249 which are catalogued as reports and K. 992 which is described as a note on private affairs. $83-1-18,45$ is also called a letter or report. These designations, in so far as they are applicable, present no fundamental distinctions; and no definition of a cuneiform letter has been established which would exclude any of these documents.

Certain general features, however, may deserve a word of comment at this point. KK. $471,884,4786,8379 ; 79-7-8,293 ; 83-1-18$, $1,53,121,125,361$; Bu. 89-4-26, 4 and probably $83-1-18,249$ are addressed to the king. $83-1-18,361$ and $\mathrm{Bu}$. 89-4-26, 4 have the usual introductory formula in such cases, ana šarri bêlia arduka, etc. $83-1-18,1$ and K. 471 use the form ana šarri mâtâti, etc. K. 8379 begins [arduka $\mathrm{m}$ il ]Marduk-apal-iddina ana dinân šarri bêlia lullik umma, cf. H. 422, 542, 781, 803, 805, $832,833,835-37,844,899,900,913,925,930,994,999,1016,1027$, 1047, 1072, and 1095. The introductory formula has been broken away in K. 4786, 83-1-18, 53, 121, $125.83-1-18,361$ places the formula of greeting to the king at the end as a colophon separated from the rest of the letter by an uninscribed space, as indicated in the text.

The name of the scribe has been preserved in only three cases, viz.: K. 8379, Mardukapaliddina; 83-1-18, 361, Ašurhamatia, and 
K. 471 which is from the citizens of Ur. The writer is unnamed in K. 884, 83-1-18, 249, 81-2-4, 48 and 83-1-18, 199, although the author's identity is otherwise determinable from the context except in the case of the last two which are strictly anonymous.

83-1-18, 1 obv. 3-8 and rev. $6-7$ contains three distinct omens.

$81-2-4,48$ and $83-1-18,199$, though described in the catalogue as letters, are in reality oracular prayers which may have belonged to a series similar to Knudtzon's Gebeten an den Sonnengott. The obverse of the former was copied by Bezold, Catalogue I, 1756, but by a comparison with $83-1-18,199$ I was able to correct his reading er-bi, obv. 3 , to si-bi. $83-1-18,199$ puts the reading si-bi beyond doubt since it will be observed that the obverse of both texts are duplicates as far as ippušu in line 6 and again in the reverse, the last two lines beginning with ezib.

We have then two prayers addressed to the same divinity concerning a reported uprising against Ašurbanipal. The oracular character of these prayers appears in the form of the question in each case. In the $81-2-4,48$, the questioner asks first, Is this uprising in the divine plan of things? and secondly, Will the rebels be successful and take possession? In 83-1-18, 199 the point of view is slightly modified, viz., Will the king successfully quell the uprising and will it be a hard struggle? Both texts then conclude with an ezib formula similar in intent to the ezib formulas in Kundtzon. I know of no other texts of this series.

Transliterations and translations of seven of these texts are given below with notes. The rest will appear in a separate article.

During my stay in London, I also collated a large number of Letter texts, the results of which collation were published in $A J S L$, XXVIII, No. 2, under the title "Textual Notes on the Letters of the Sargon Period." These notes are reprinted as an appendix.

I am under great obligations to Dr. E. Wallis Budge, the keeper of the Department of Egyptian and Assyrian Antiquities, British Museum, and to Mr. Leonard W. King, Mr. H. R. Hall, and Mr. P. S. P. Handcock, assistants in the department, for their courtesy and for the kind assistance which they afforded me during my stay in London. I am also under obligations to Dr. Sigurd Ylvisaker for several oral suggestions. 
Some Kouyunjik Letters and Related Texts

KOUYUNJIK LETTERS AND RELATED TEXTS

\section{1}

$89 \%-18,1$.

Obverie.

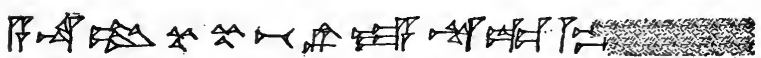

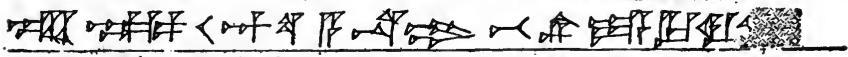

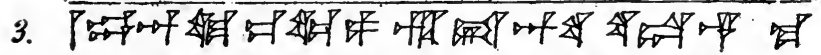

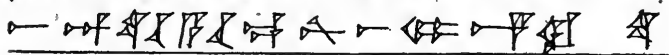

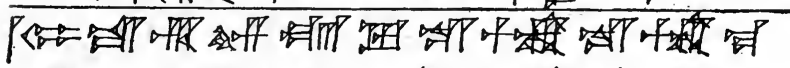

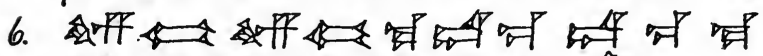

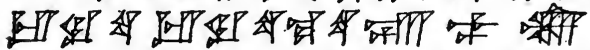

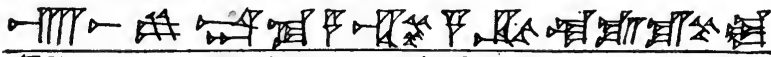

9.

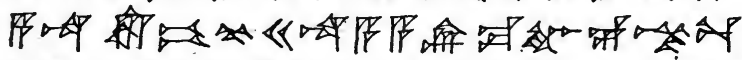

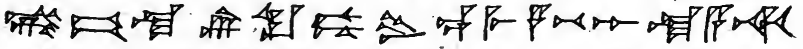

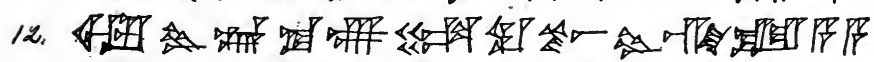

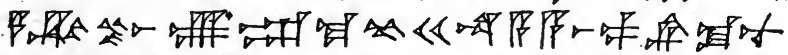
$\Rightarrow$ 罚布

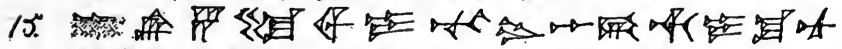

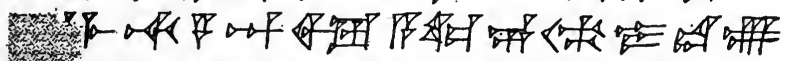

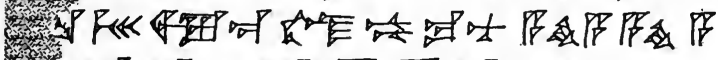

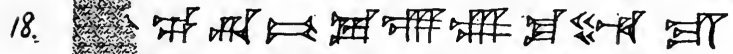

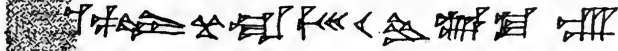

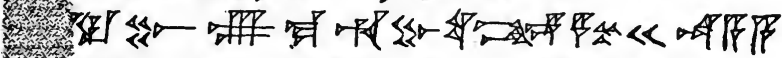

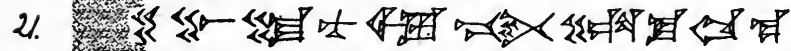
cdge.

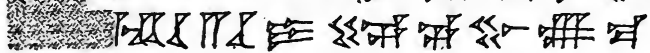




\section{1 (Continued)}

\section{3-1-18,/(continued) Reverses.}

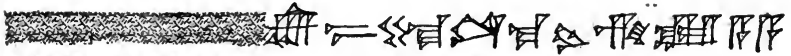

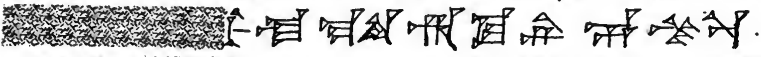

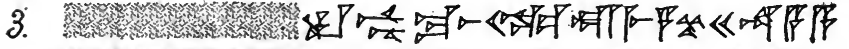

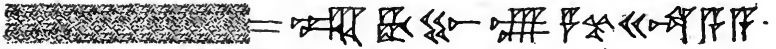

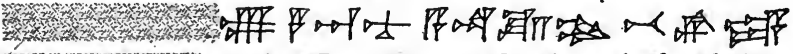

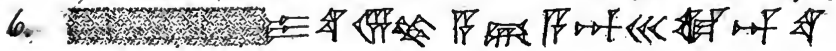

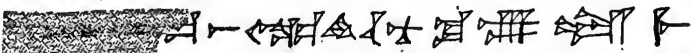

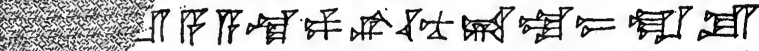

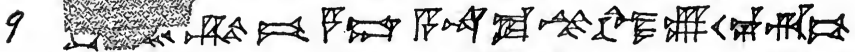

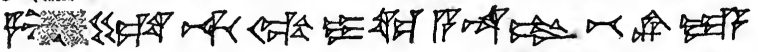

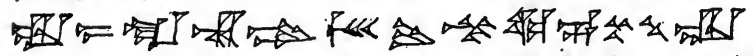

12. ¿

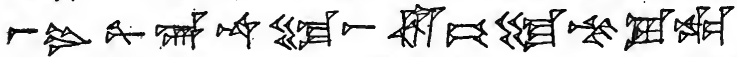

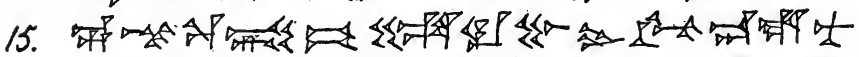

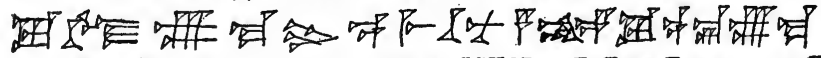

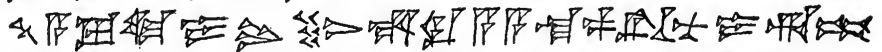

18. 形

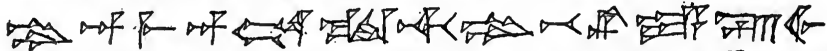

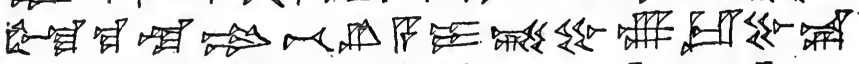

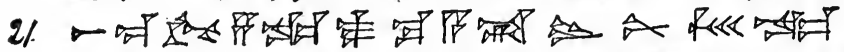

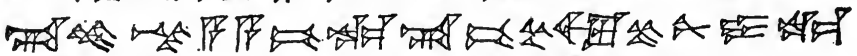

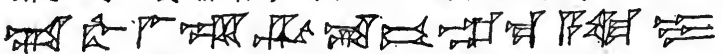

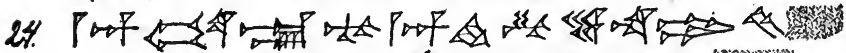

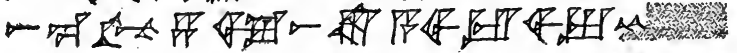

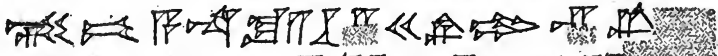

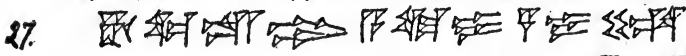


$83 \%-18,125$.

Obverse.

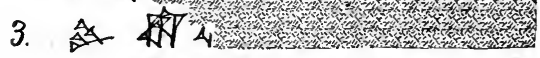

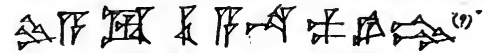

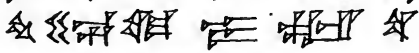

6. (exasure)

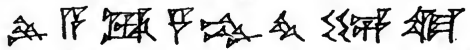

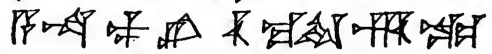

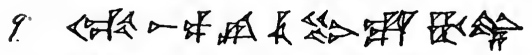

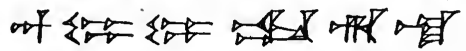

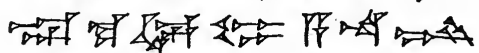

12 以

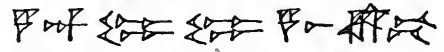

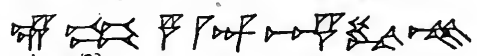

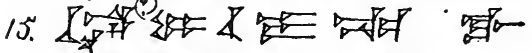

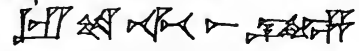

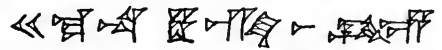

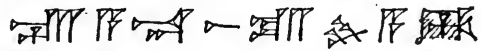

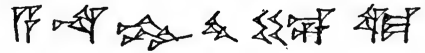

Reverse:

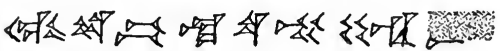

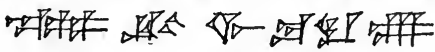

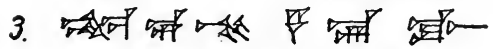

DORR

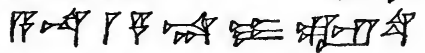

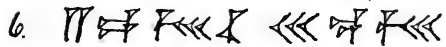

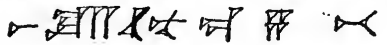

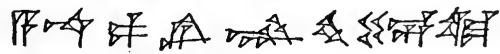

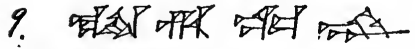

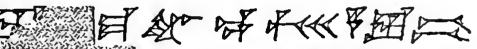

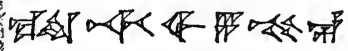

12:

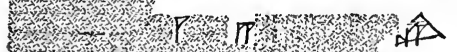

k 


$$
\begin{aligned}
& 3,4 \\
& 81-2-4,48 . \\
& \text { Obucrse. }
\end{aligned}
$$

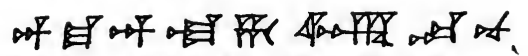

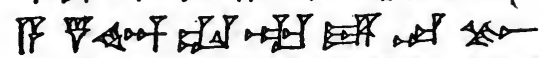

3. of

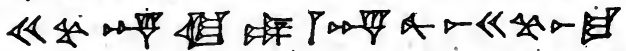
原

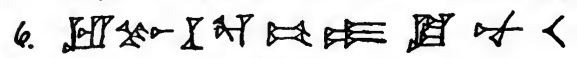
Reverse.

E N 世

3. 一ATP

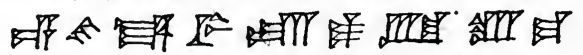

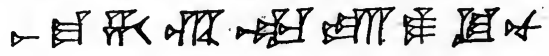

$$
\begin{aligned}
& \text { 85-1-18, } 199 . \\
& \text { Obuerse. }
\end{aligned}
$$

3.

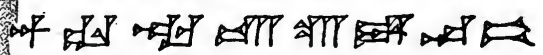

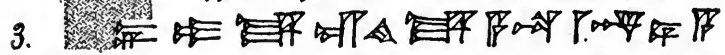

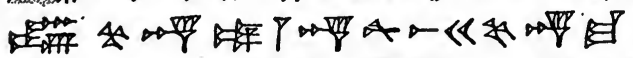
䂟会 6. IEIT Reverse.

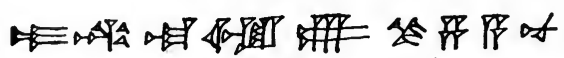

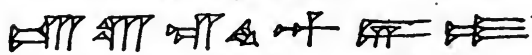

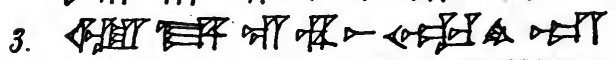

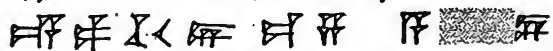

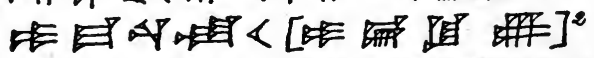

6. HP -

$$
\begin{aligned}
& \text { 'Aritten over an erasure. } \\
& \text { "Oraces almost illegible; one can } \\
& \text { ferhapes read ac abore foel roving the }
\end{aligned}
$$




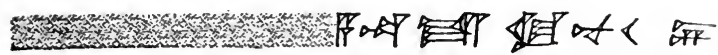

併

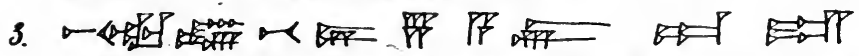

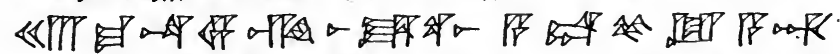
of

6.

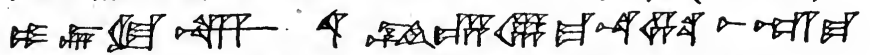
DTP 9. Tr

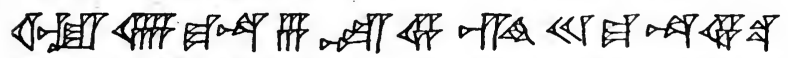
TFaA

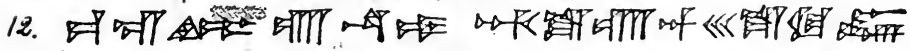
Trot \&

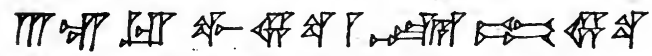
idged

If

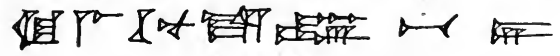
Reverse.

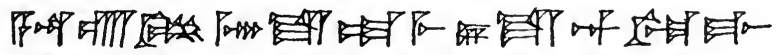

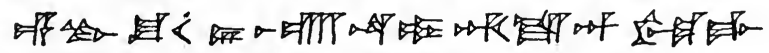

3.

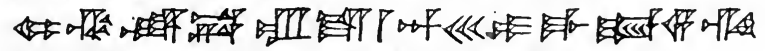

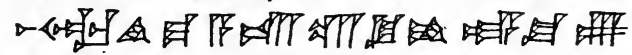

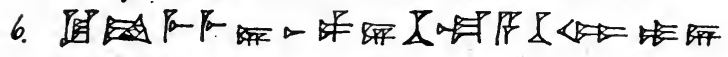

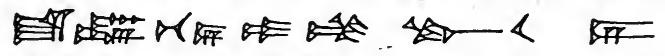

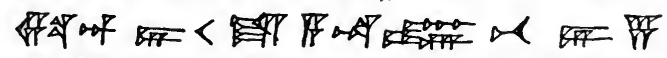

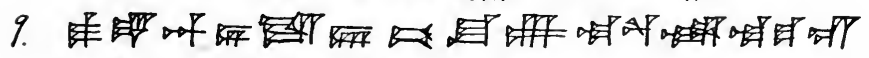

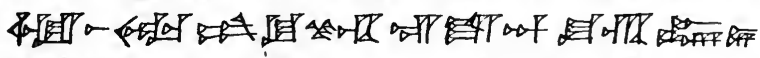

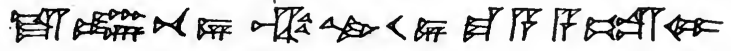

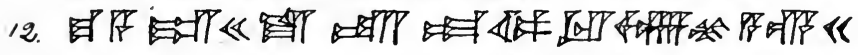

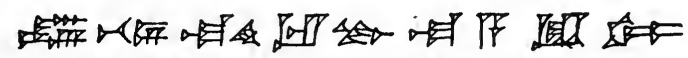

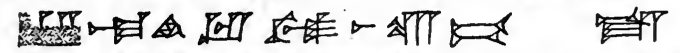


The American Journal of Semitic Languages

6

\author{
$K 4786$ \\ Obverse.
}

3. T. 2.

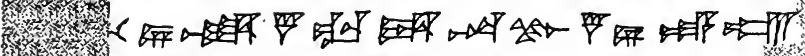

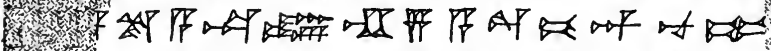

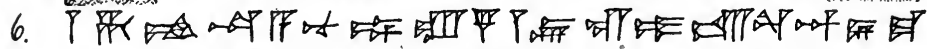

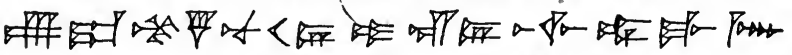
OH

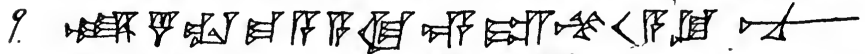

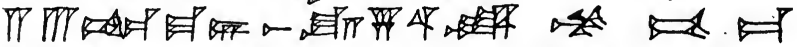

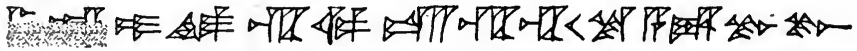
12.

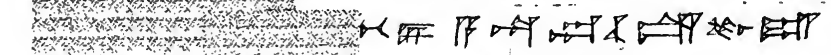

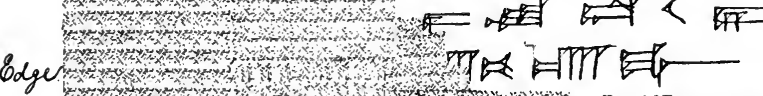

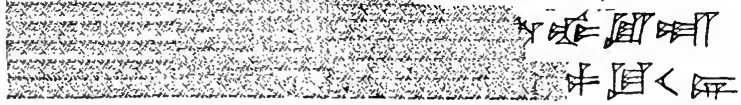
Reverse

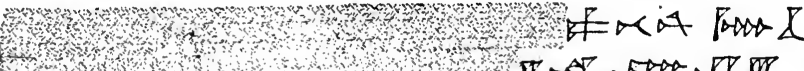 If $\rightarrow$ P

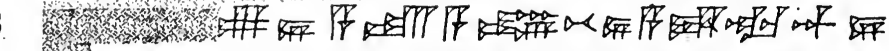

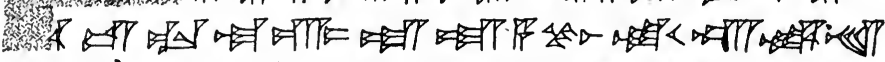

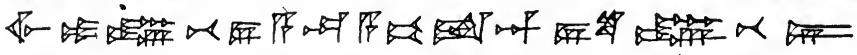

6 AाT"

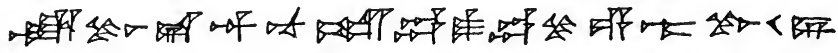

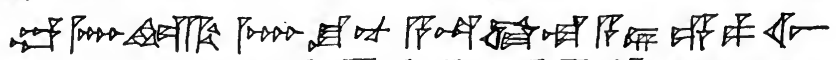

9. TाP

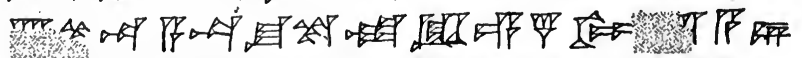

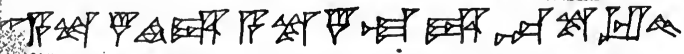

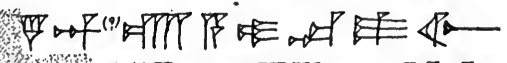

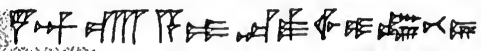

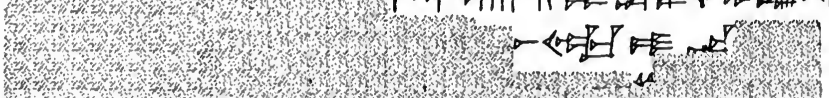




\section{7 \\ $83 \% 18,45$. \\ Obierse.}

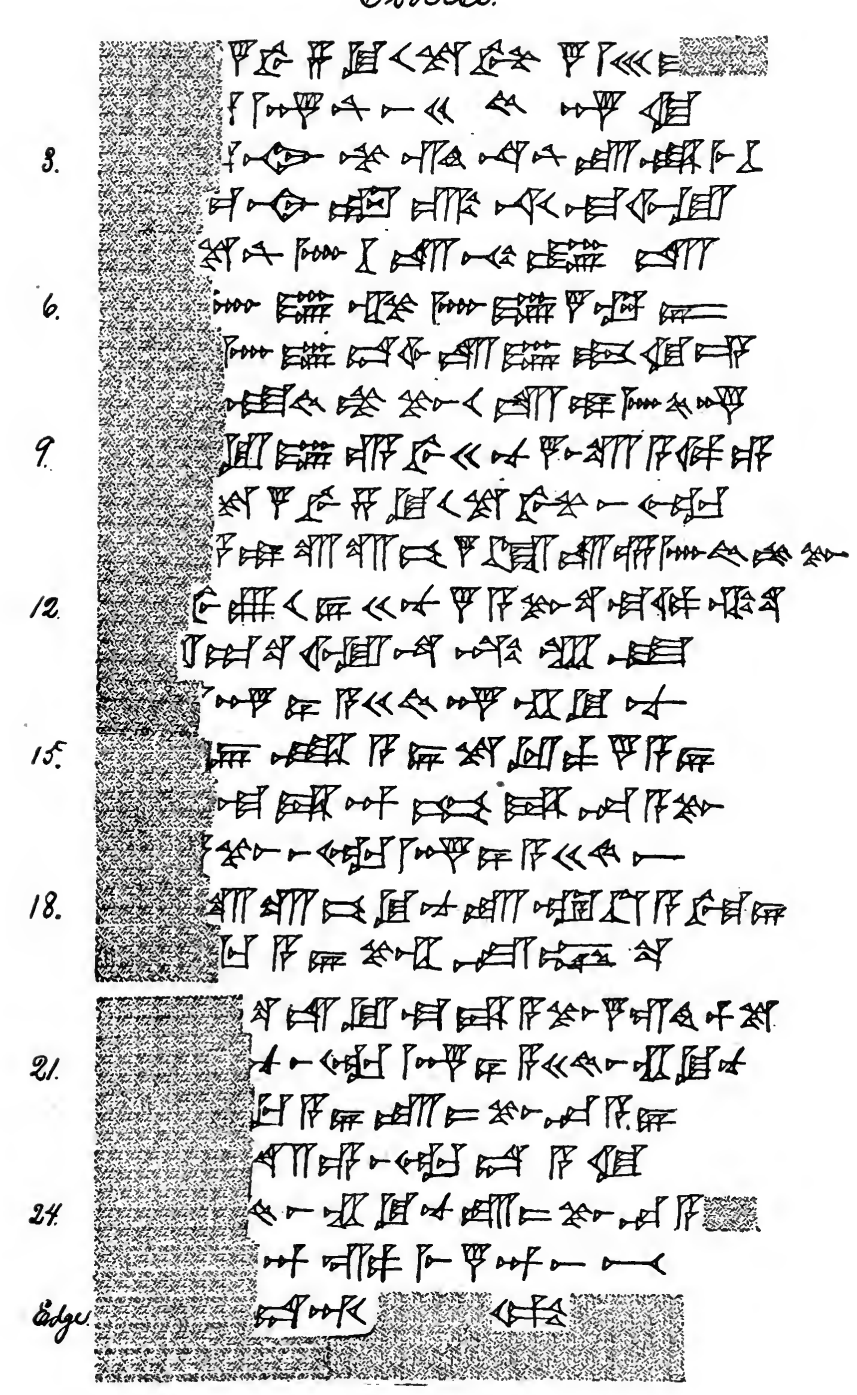


7 (Continued)

83-1-18,45(continued)

Reverve.

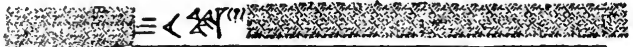

1.2.

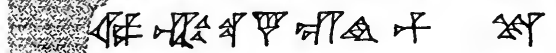

3.

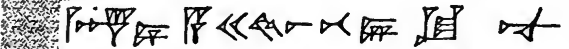

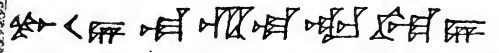

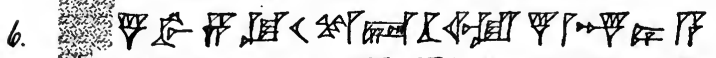

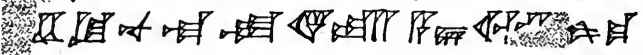

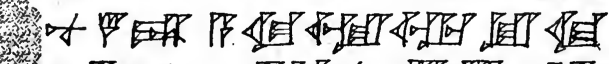

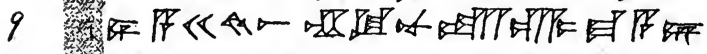

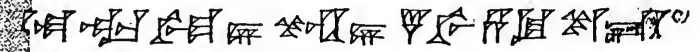

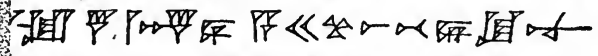

12

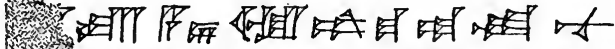

-

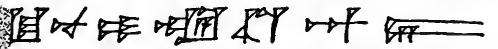

15. 約緇

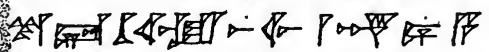

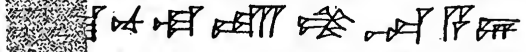

18.

1.

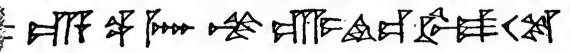

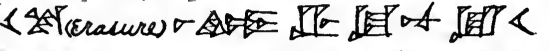

21.

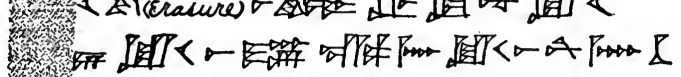

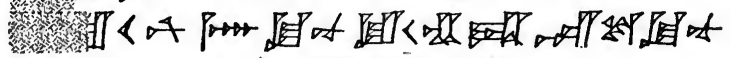

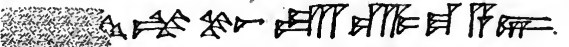

2.4.

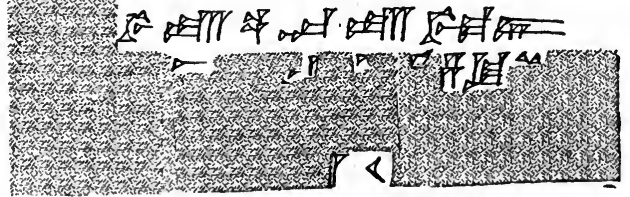


Obocrue.

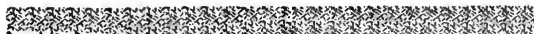

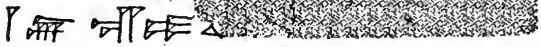

3. 细进 大

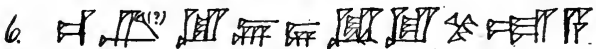

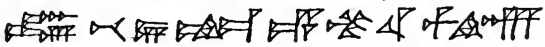
值目会

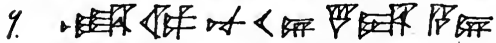

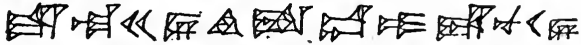

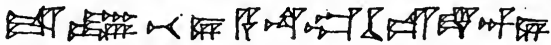

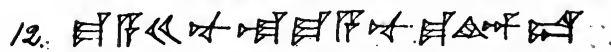

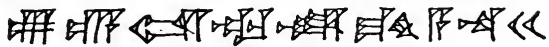

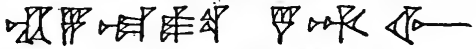

15.

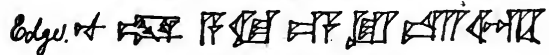

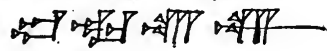
Reveras.

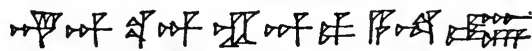

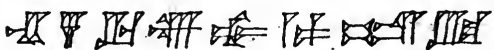

3. एक 相 rATP

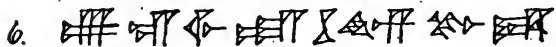

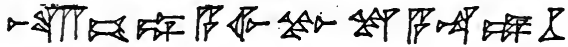

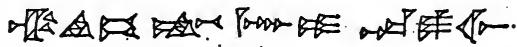

9 व

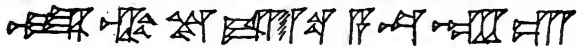
E

12. の

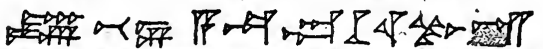

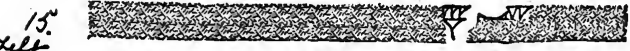

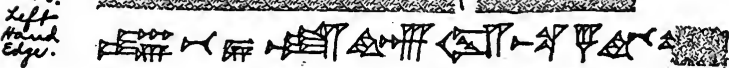

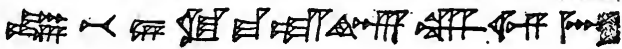


$83-1-18,361$.

Obverse.

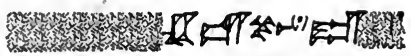

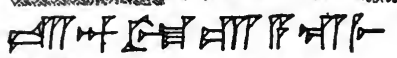

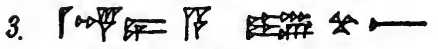

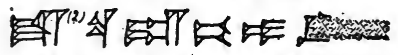

经

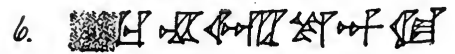

铰语

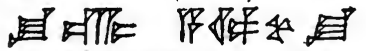

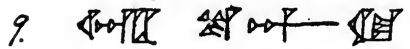

- सा

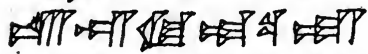

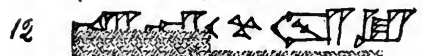

1.

Reverse.

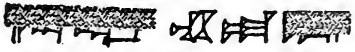

W नाय नाय

3. Port《计

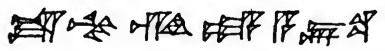

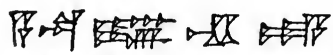

6. 엄

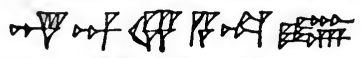

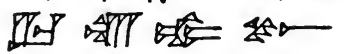




\section{Que 89.4-26, 4 Obverses}

度

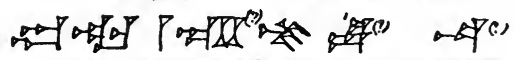

3.

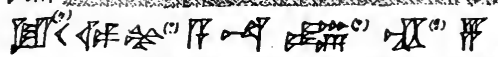

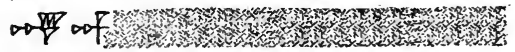

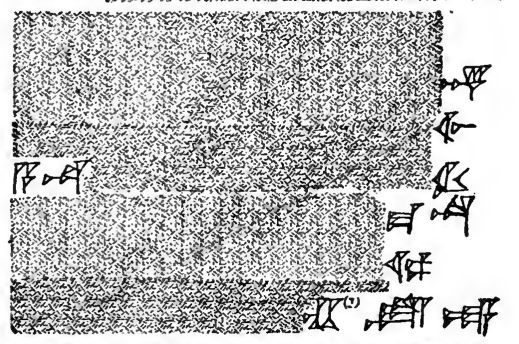

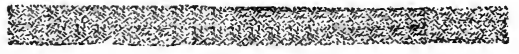
Reverse.

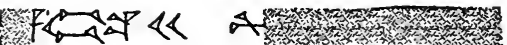
僜

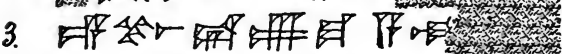
促"

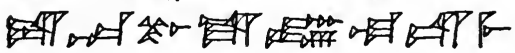

6. Ap

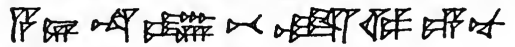
DP

9.

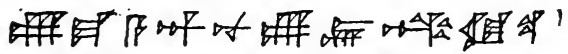

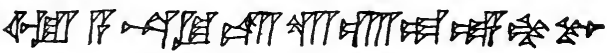

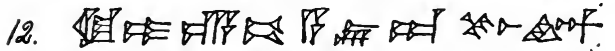
ज्ञा ए

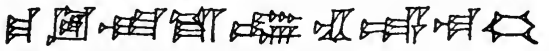

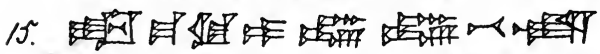

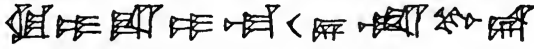

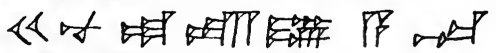

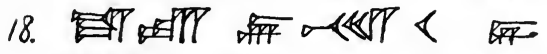

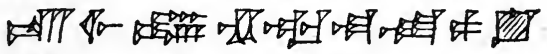

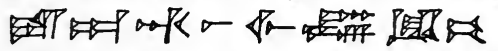

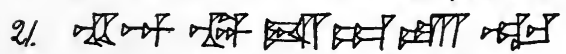




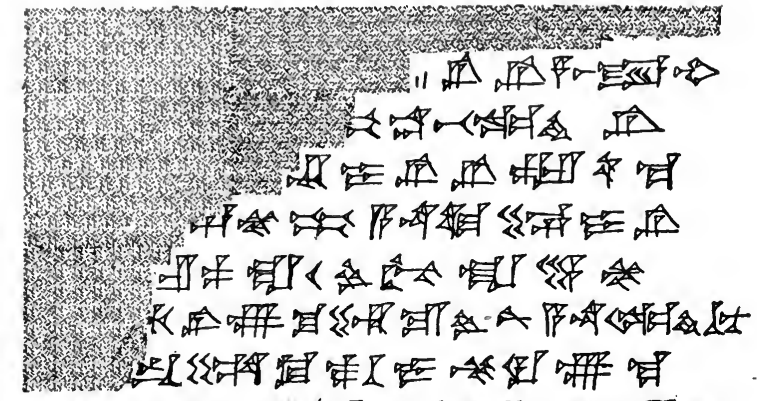

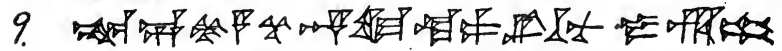

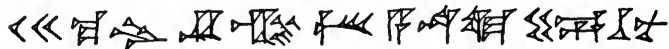

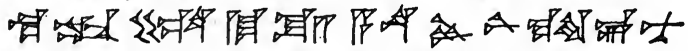

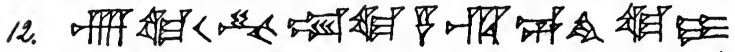

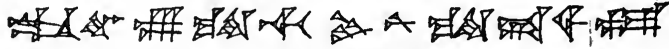

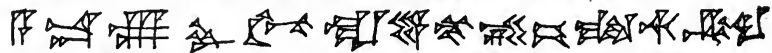

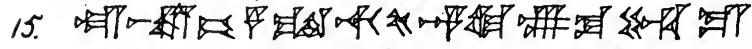

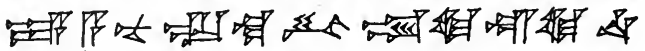

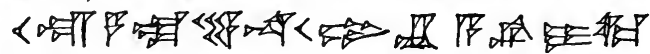

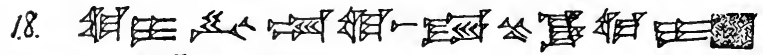
Edge.

\section{Reverse.}

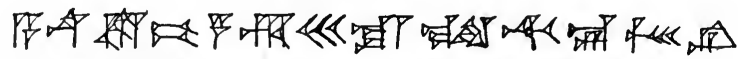

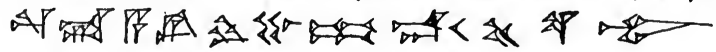

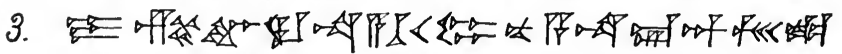

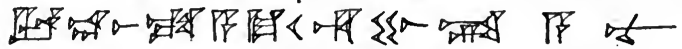

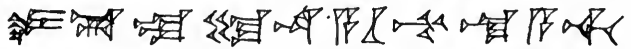

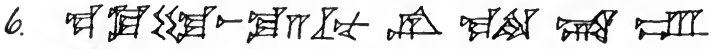

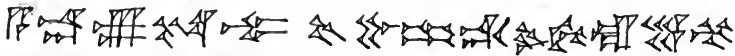

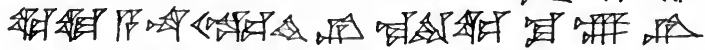

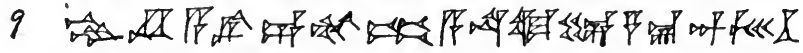

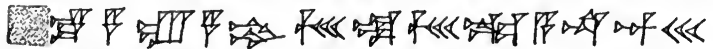

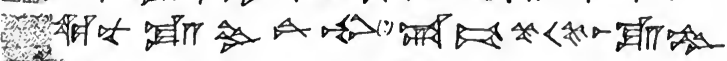

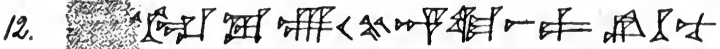

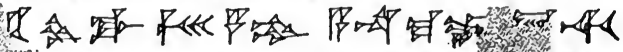




\section{2 \\ K.47/. \\ Obverse.}

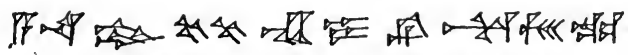

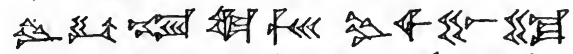

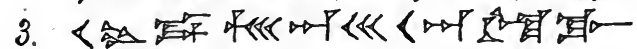

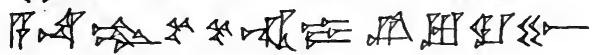

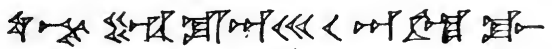

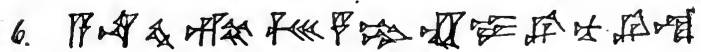

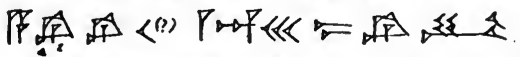

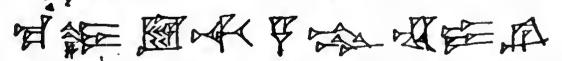

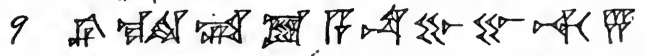

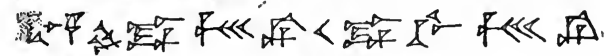

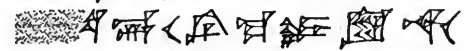

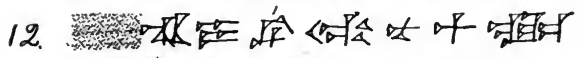

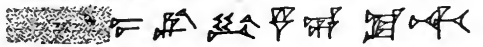

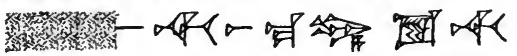

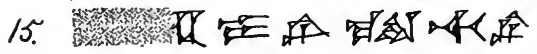

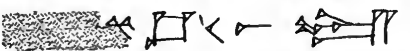

10.

18.

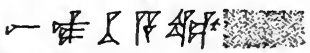

\&dgex

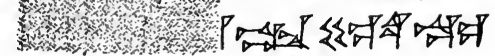

Reverse.

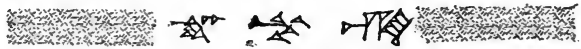

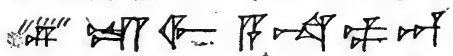

3 ए人民地

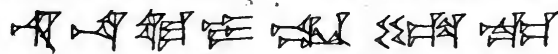

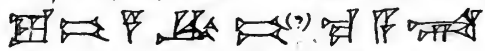

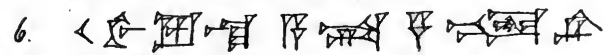

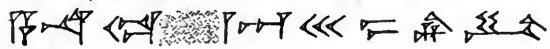

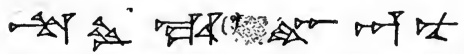

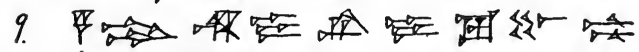

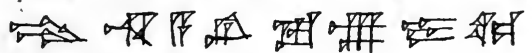


13, 14

Kils45.

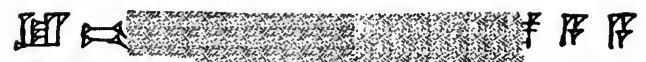
$\Delta \mathbb{H}^{\circ} \circ \mathrm{OP}^{2}$ -

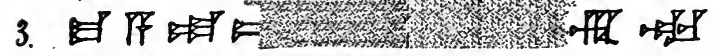

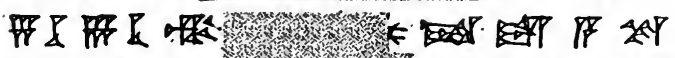

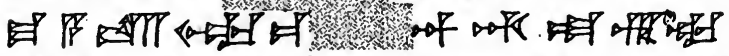

6. 4 UIP

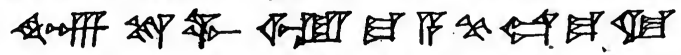

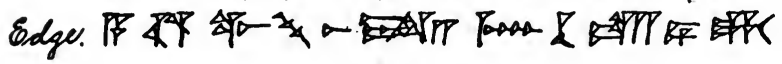
Rescrese.

.

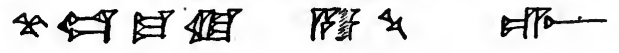

K. 992.

Obverce.

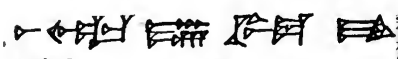

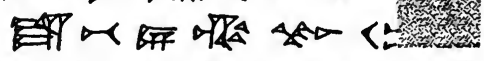

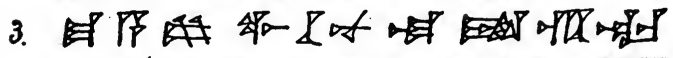

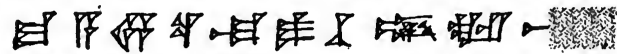

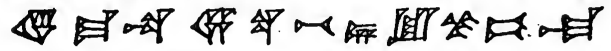

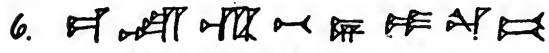

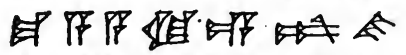

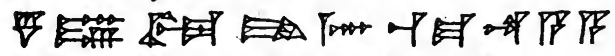

9. एक

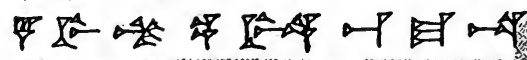
नाR

12

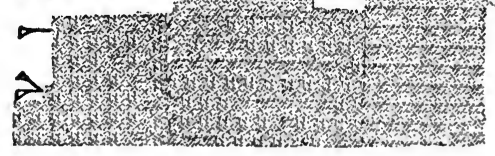




\section{4,15}

$$
\begin{aligned}
& \text { K.992(continued) } \\
& \text { Beverse. }
\end{aligned}
$$

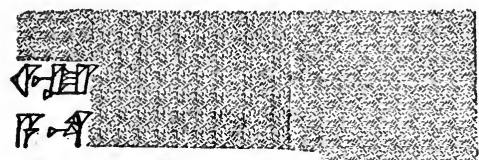

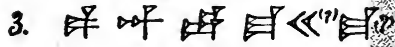

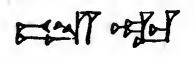

$83-1-18,249$.

Oborese.

-A A

$$
\text { 佊然四 }
$$

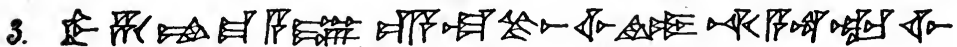

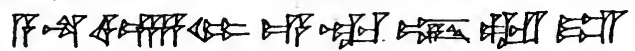

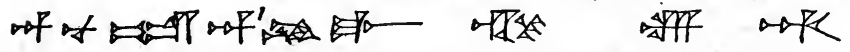

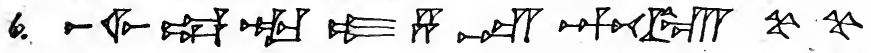

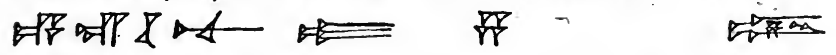
AाT or

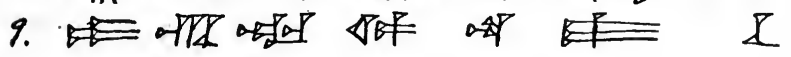
Reverse.

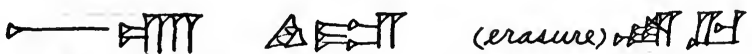
or 荡

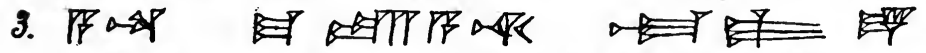

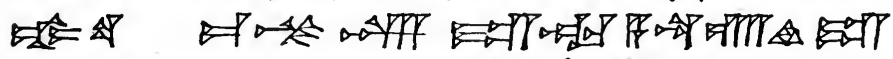
पर्या

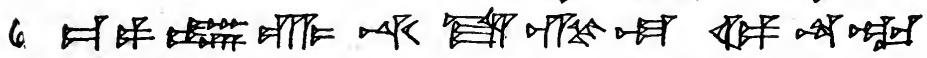
ST

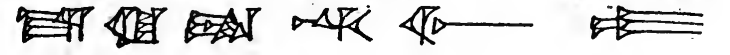


K. 88.4

Oborese.

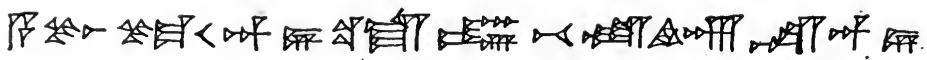
IET D A

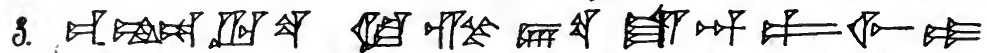

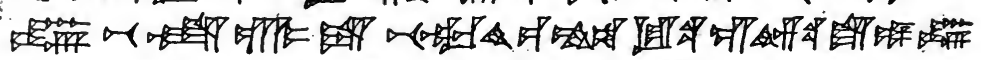
叶的

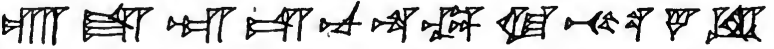

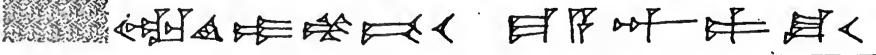

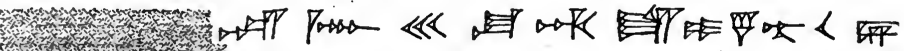

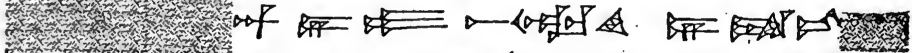
算:

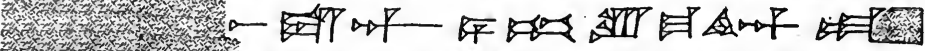

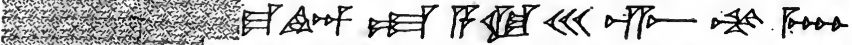

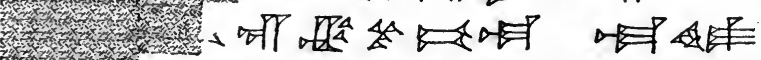

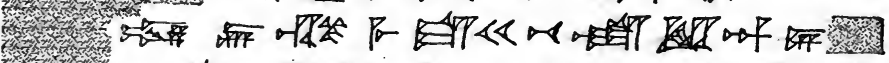

15.

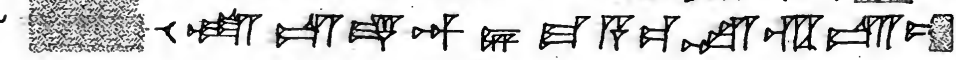
Reverse.

1.

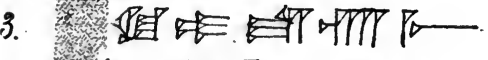

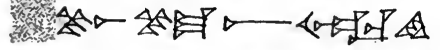

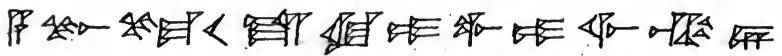

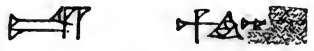
or A. AT $<$ ए AT

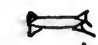
Fit

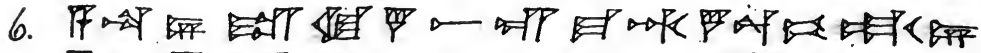

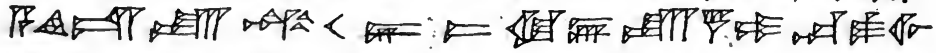
展口会

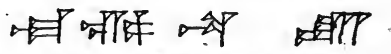

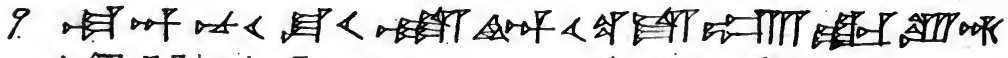

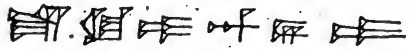
एक्षात Tror

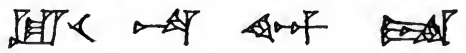




\section{7}

K.8379.

Obverse.

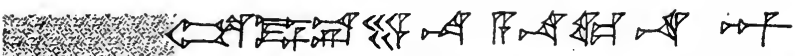

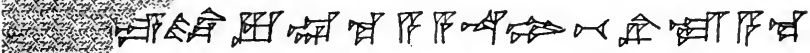

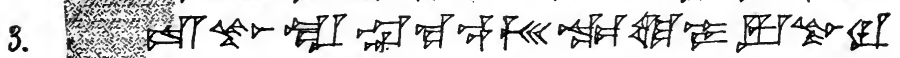

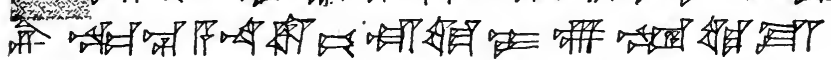

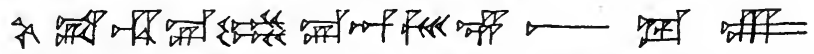

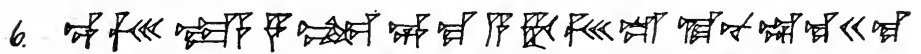

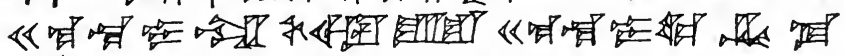

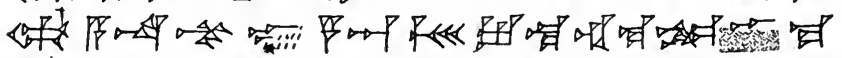

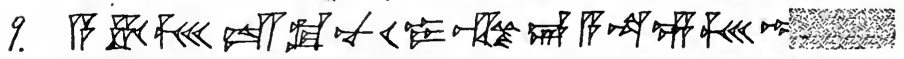

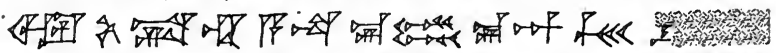

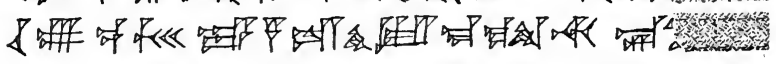

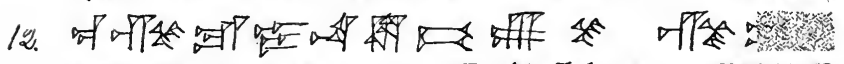

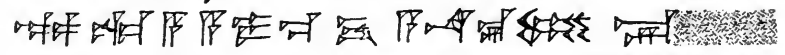

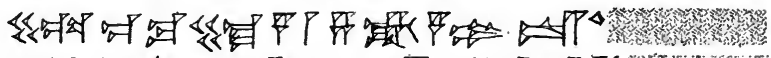

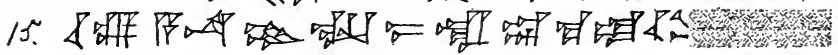

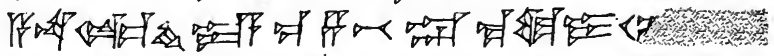

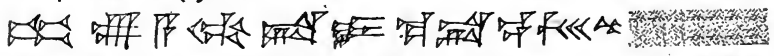

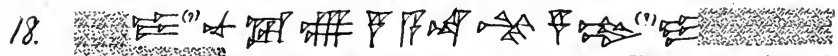

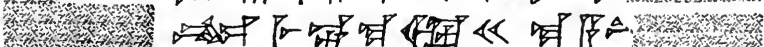

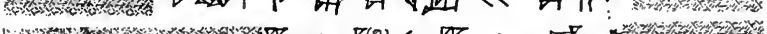

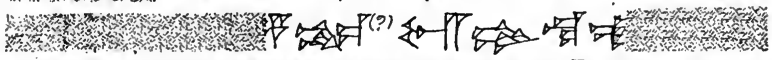

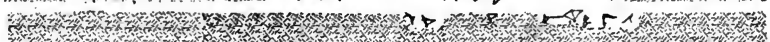

\section{Reverse.}

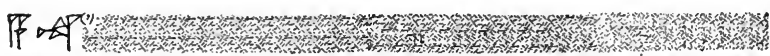

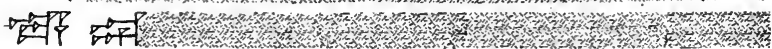

3. 1 年

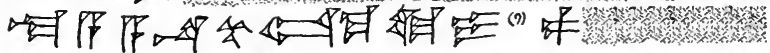
P 
1. $83-1-18,1$

OBVERSE

A-na šar mâtâti be-lí-ia ardu-ka m [. . . . . . .

ilBêl il Nabû u ilšamaš a-na šarri be-lí-ia lik-ru-[bu]

3. Ana kakkabšir'û ișți-pa-ri ištu sît šamši iṣrur-ma ina erêb šamši irbi ummâni nakri ina mi-til-ša šumkût(ut)

Ana mûši ša-ri šûtu iš-kun iš-kun-ma

6. im-șur im-șur-ma izziz(iz) izziz(iz)-ma ip-ru-ud ip-ru-ud-ma u-sa-pi-ib rubû ina ḥarrâni illaku(ku) mimma šumšu bušû ḳât-su ikaššad(ad)

9. ki-i šarru a-na e-mu-ḳi-šu il-tap-ru um-ma a-na lib-bi mât Man-na-a-a ir-ba-'a e-mu-ḳa gab-bi la ir-ru-ub amêl șâbê ša bít-bal-la-a-ti

12. ù amêlDak-ku-ú li-ru-bu amêl Gi-mir-a-a

ša iḳ-bu-ú um-ma mât Man-na-a-a ina pa-ni-ku-nu šêp-a-ni ni-ip-ta-ra-su min-di-e-ma

15. . . . . . ir ía tu Š̀I i zêr amêl bal-ga-ti-i-šú-nu

[ma-a]m-me-ti ša ili ù a-di-e ûl i-du-ú

[is. narka]bâti ù iṣ șu-ub-ba-nu a-bi-a a-bi-a

18. [ša ni]-e-ri-bi lu-ú ú-šú-uz-zu

[a-d]i sisê u amêlDak-ku-ú

[li]-ru-bu-ú-ma bu-bu-ut bilti ša mâtMan-na-a-a

21. [li-]hi-bu-tu-nu u lil-li-ku-nim-ma

\section{EDGE}

. . . . ni-e-ri-bi lu-ú ú-šú-uz-zu

. . . . . . ištên-šu šani-šu i-tir-e-bu-ú-ma

REVERSE

. . . . . . . . i]b-tab-tu-nim-ma amêlGi-mir-a-a

. . . . . . . la it-tal-ku-ni e-mu-ka

3. . . . . . . ir]-ru-ub-ba ina mubhbi alâni ša mâtMan-na-a-a

. . . . . . . ilBêl-ha-pu-ú ša mât Man-na-a-a

. . . . . . . ú-ša-an-nu a-na kâati šarri be-lí-ia

6. . . . . . . . i ûmi XVkan a-ga-a ilšin itti ilšamši

[in-nam-r]u ina mub-bi-šu-nu šú-ú šêpâ(me)

[amêlGi-mi]r-a-a la-pa-ni-šu-nu ta-at-tap-ra-su

9. [šum-]mu ik-kaš-ša-du a-na-ku mu-șu-ú u e-ri-bi ša . . . . . li-ti ûl i-di a-na šarri be-lí-ia al-tap-ra bêl šarrâni amêli mu-di-e mâti liš-al

12. ù šarru a-ki-i ša i-li-'i-ú a-na e-mu-ḳi-šu liš-pur mu-uš-ta-bal-ku-ti ina mubbi mun-dah-ṣu-ti ina amêl nakri dan-na-tu ina lib-bi tu-mu-lu-ka 
15. e-mu-ka gab-bi li-ru-bu amêl Gu-du-da-nu

lu-ṣu-ú-ma amêl șâbê (me) -šu-nu ša bilti lu-ṣab-bit-ú-ma

liš-a-lu ki-i amêl In-da-ru-a-a la-pa-ni-šu-nu i-ri-ḳu

18. e-mu-ḳu li-ru-ub ina muhbi alâni lid-du-ú

šar ilâni il Marduk it-ti šarri be-lí-ia sa-lim

mimma ma-la šarru be-lí-a i-ḳab-bu-ú ip-pu-uš

21. ina iṣ kussi-ka aš-ba-ta amêlnakrê(meš)-ka

ta-kam-mu a-a-bi-ka ta-kaš-šad ù mât nakri-i-ka

ta-šal-lal ilBêl ike-ta-bi um-ma a-ki-i

24. m il Marduk-šâpik-zêri m il Ašur-ah-iddin-na šar mât[Ašur . . . . .

ina iṣ kussi ù ina libbi a-ši-ib ù m[âti . . .

gab-bi a-na ḳâti-šu za( ?)-man-ni šarri bêli-[a i-di]

27. ba-di-iš šarru a-ki-i ša i-li-['i-ú]

$$
\text { li-pu-uš }
$$

(Obv.) To the king of the lands, my lord. Thy servant . . . . May Bêl, Nabû and Šamaš be gracious to the king, my lord.

When the constellation Virgo shines forth from the sunrise like a torch and in the sunset fades away, the troops of the enemy will be severely smitten.

When the south wind blows all night, and having blown all night, (still) continues, and as it continues becomes a gale; and from a gale increases to a tempest; and as a tempest does sweeping damage; the prince on whatever expedition he goes, will obtain wealth.

Whereas the king has sent (an order) to his army, as follows: "Enter into the midst of the Mannai." The whole army (I assume) is not to enter. Let the cavalry and the Dakkû invade the Gimirai, who made the declaration, saying: "The Mannai pertain to you, we shall not interfere." Certainly this is a lie(?). The offspring of their fugitives recognize neither the oath of a god nor a (human) agreement. Let the chariots and baggage wagons take up a position on either side of the entrance; (then) with the horses and the Dakkû, let them enter and plunder the produce of the Mannai; and let them return and at the entrance let them bivouac .... once or twice they entered and (Rev.) . . . . plundered and the Gimirai .... they came, the army .... shall enter against the cities of the Mannai . . . Bêl-hapû of the Mannai . . . . will change. To the hands of the king my lord . . . . on the fifteenth day the full moon 
appeared with the sun. This is against them. Wilt thou be hindered by the Gimirai? If they approach, their comings and goings . . . I I do not know. I have sent a message to the king, my lord. May the lord of kings inquire of a man acquainted with the country and may the king, at his pleasure, send to his army raiders in addition to the other fighting men. A fortress in the midst of the enemy's country do thou provision for thyself. Let the whole army enter it. Let the Gududanu go forth and let their soldiers seize upon the produce and let them inquire whether the Indaru'ai have departed. Let the army enter against their cities. Let them overthrow them.

The king of the gods, Marduk, turns graciously to the king, my lord. Whatever the king, my lord, asks, he will grant. Upon thy throne thou art seated, thine enemies thou shalt take captive, thy foes thou shalt conquer, and the land of thine enemies thou shalt despoil. Bêl has spoken, saying: how Mardukšapikzêri Esarhaddon, king of Assyria .... upon the throne and he is (now) seated thereon and how the whole land (is) hostile to his rule. The king, my lord, knows. Joyfully let the king do according to his pleasure.

In spite of the fact that the name of the scribe is missing and the reigning king unnamed, the historical and political situation in this letter is fairly clear. The land of the Mannai, bordering on Lake Van, had practically been an Assyrian protectorate since the days of Tiglathpileser I. The people rebelled under Sargon and received cruel punishment. Under Sennacherib, the Gimirai appear and, joining with the Mannai, they developed a situation almost identical with that represented in the present text. That it was not the same occasion is to be inferred from rev. 24, where Esarhaddon seems to be mentioned as a previous king. This would bring our text down to the reign of Ašurbânipal (668-626); and from the annals we know that there was a revolt among the Mannai during his reign. One of his campaigns due to Mannean agressiveness is referred to in Knudtzon, No. 150.

The writer was evidently a military official of high rank, who enjoyed the close confidence of the king. It is noteworthy to observe such an official advising the monarch and planning campaigns that modify the king's order, and also go far beyond it. We know that the Rab-šak, who made a campaign against the Mannai, was Nabûšarușur, also called Rab-mugi (cf. Klauber, $52^{2}$ ). We also know of an important campaign intrusted to him against the Gambulai in the southeast, and that he was sent on diplomatic missions to the city of Arvad and the land of Musri, cf. Knudtzon, Nos. 66, 67,150 , and 153. The traces of the name of the scribe in obv. 1 lend themselves to the above officer's name. 
Obv. 1. On the use of šar mâtâti as an indication of the reign, ef. Behrens, $60^{2}$.

Obv. 3. For the identification MUL.KI.=šir' $\hat{u}$, also abšênu lit. "grain in the ear," corresponding to Virgo, cf. Jensen, 311; on the origin and significance of the name, ibid., $500 \mathrm{f}$. For this use of SUR, i.e., iṣrur, cf. Thompson, No. 28, rev. 2, note.

Obv. 4. AN.UD.ŠU.A., i.e., erêb šamši, Br. 10828. In the reading mi-til-ša, the sign til (AŠ) touches the ša following, but the difference between this possible SUR and SUR in the line above is to be noted. Besides, SUR makes no sense here while mi-til-ša, lit. "its power," has a parallel construction in mi k -ti-ša. Cf. Thompson, No. 187, rev. 7. Our form is perhaps to be supplied in Thompson, $246 E$, obv. 2. RU+u t, i.e., šu mkût III ${ }^{1} \mathrm{pm}$. of בעקת. For this form in omens, cf. Thompson, 44, rev. 8, 50, obv. 2. For the general form of this omen, cf. Thompson, 187, rev. 5-7. The omen itself consists in the sun's having reached the zodiacal sign Virgo.

Obv. 5. Lit. "when the south wind has established a night of wind," i.e., when it has blown all night.

Obv. 6. imșur I take as denominative of mașartu, cf. HWB. 423a, lit. "kept the watch," and so "continued." DU+i i, i.e., izziz, lit. "stood," I take as part of a description of the wind's increasing force, "stand" being used in the sense of "be firm, steady," which, taken with what precedes, I have rendered by "gale."

Obv. 8. ṠA.NAM, i.e., mimma šumšu, Br. 12009. ŠA.GAL, i.e., bušû Br. 12015.

Obv. 10. irba' impt. of ארב ; cf. ipša', H. 401, obv. 13; alka' H. 459 , obv. 2.

Obv. 11. ZAB. ${ }^{\mathrm{m} e}$, i.e., șâbê, for other cases of me for meš in this text, cf. rev. $3,8,16,18$, and 19 . On bít-bal-la-a-ti, a fem.pl., cf. $B A S$, I, 211, and $A J S L, \mathrm{XI}, 198$.

Obv. 13. in a pa-ni-ku-nu, lit. "in front of you."

Obv. 14. mindiema, cf. Johnston, JAOS, XVIII, 148, "when, if"; Figulla, 52, "vielleicht"; Ylvisaker, 64 f., "sicherlich." šêpâni niptarasu, lit. "our feet we shall restrain." I would regard this as a relative clause with the ša omitted.

Obv. 15. There are two uncertainties in this line that materially affect the context. The first sign may be almost anything and the third sign may be sa a as I first copied it, but following the eye I have written it ia. With much reservation I would supply pi as the first sign and read s a instead of ía, regarding the latter as due to an accidental scratch or a scribal error, transliterating pi-ir-ṣa-tu ši-i zêr amêlhal-ga-ti-i-šu-nu and translating as above.

Obv. 16. The upright stroke in a $\mathrm{m}$ of mamêti may be the sixteenth of an inch too far to the right; otherwise $[\mathrm{ma}-\mathrm{a}] \mathrm{m}-\mathrm{me}-\mathrm{ti}$ suits the space requirements. 
Obv. 17. The ideogram for narkabtu seems fairly certain from the spacing, the traces, and the context. ișs u-ub-ba-nu I would regard as an unusual pl. for the more normal șumbê(-âti], Del., Gram., § 93a.2. Cf. a-pa-a-ni for a pâte, têmâni for têmâti, Ylvisaker, $\S 20$.

Obv. 18. On the form ušuzzu cf. Ylvisaker, $38 .^{4}$

Obv. 21. For the form li-hi-bu-tu-nu for lih-bu-tu-nu and for other examples, cf. Ylvisaker, $\$ 8$.

Edge 2. i-tir-e-bu-ú-ma for it êrbûma $I^{2}$ of =רא.

Rev. 1-6 are too badly broken to admit of restorations. The general thought, however, seems to be that the result of the campaign as planned by the writer would be the restoration of the Mannean cities to the king of Assyria.

Rev. 6-7 consist of a third omen, although not set off by lines as are the first two. For this class of omens, cf. Thompson, 153, 156.

Rev. 7. A verb is required at the beginning of the line and innamru corresponds to the usual form and suits the traces.

Rev. 8. ta-at-tap-ra-su IV of פרפ. I would regard the overhanging vowel as interrogative.

Rev. 9. The traces favor the reading šum-mu; for other cases of šummu for šumma in the letters, cf. H. 25, 99, 101, 138, 378, 389, 453, 556.

Rev. 10. The first two signs are quite uncertain.

Rev. 13. mu-uš-ta-hal-ku-ti, III ${ }^{2}$ ptc. pl.; mun-dab-șu-ti I ${ }^{2}$ ptc. pl., both used substantively.

Rev. 14. tu-mu-lu-ka for tu-mal-li-ka II ${ }^{1}$ of $N^{2}-2$; for the $u$ vowel before $\mathrm{lu}$, cf. Ylvisaker, $\S 7 c$.

Rev. 26. za(?)man-ni I would take for zamâni. Cf. Del., Gram., $\S 48$. However, the equally possible reading a-man-ni, prs. of 79 , is supported by a suggestion from Dr. Ylvisaker.

\section{3. $81-2-4,48$}

OBVERSE

ilu ma-an la-ha-ar ba-nu

a-ša-'a-al-ka da-ba-bu

3. an-ni-i šá si-hi šá a-na mAšur-bân-apli

šar mât Ašurki mâr mAšur-ah-iddina šar mât Ašur-ma

iḳ-bu-u-ni ma-a si-hu ina mub-hi ali

6. ip-pu-šu ḳa-bi-i ku-nu-u

i-ba-aš-ši i-sah-tu-u-ni(?)

REVERSE

i-kaš-ša-du-ni-i a-mu-a-ta

3. ina lib-bi i-sab-ba-tu-ni-i

e-zib ša sinništu ta-aš-țu-ru-ma

ina ma-ba-ri-ka ta-aš-ku-nu 
4. $83-1-18,199$

OBVERSE

[ilu ma]-an la-ba-ar ba-nu-ni

[a-ša-'a]-al-ka ultu lịbbi da-ba-bi

3. [an]-ni-i šá si-bi šá a-na $m_{A}$ šur-bân-apli

šar mât Ašur mâr mAšur-ah-iddina šar mât Ašur-ma

ike-bu-u-ni ma-a si-bu ina mub-bi ali

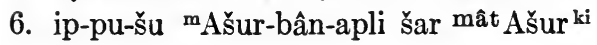

REVERSE

i-bal-la ù ú-še-șa-a-nu

ultu libbi si-bi an-ni-i

3. ù šá si-bu ina muh-bi ali

e-pa-šu-u-ni iz-za-a[-zu-]ni

i-ma-ka-tu-u [i-dak-ku-u]

6. e-zib šá sinništu ta-aš-[țu-ru-] ma

ina ma-ba-ri-ka taš-ku-nu-u.

\section{3}

(Obv.) O god, whomsoever it is good that I supplicate, I beseech thee concerning that matter of the revolt which they have reported to Ašurbânipal, king of Assyria, son of Esarhaddon, king of Assyria also, as follows: They are stirring up a revolt against the city. Is it decreed? Is it established? (Rev.) Will it come to pass? Will they take the aggressive? Will they conquer? Shall I die? Therein will they take possession? Heed not that a woman has written this and placed it before thee.

\section{4}

(Obv.) O god, whomsoever it is good that I supplicate, I beseech thee concerning that matter of the revolt which they have reported to Ašurbânipal, king of Assyria, son of Esarhaddon, king of Assyria also, as follows: They are stirring up a revolt against the city. (Rev.) Will Ašurbânipal, king of the land of Assyria, conquer and drive them forth from the midst of that revolt? And as for those who are stirring up the revolt against the city, will they stand? Will they fall? Will they kill? Heed not that a woman has written this and placed it before thee.

Concerning these two texts in general, compare the introduction. For the above translation of obv. 1, I am indebted to a kindly personal note from Professor Christopher Johnston. He would regard ma-an as a short form 
of man-nu, la-har for lah-har, as a precative of $7-1 / 2$ and ban $\hat{u}(\mathrm{ni})$ as a pm. This rendering makes of these texts general formulae for enquiring of any god.

3, obv. 6. ka-bi-i ku-nu-u, both pms.; for the formula, cf. Knudtzon, p. 23.

3, rev. 1. i-sah-tu-u-ni(?) I² of lit. rage; or shall we read $\mathrm{i}-\mathrm{ba}-\mathrm{a}$ š-ši-i kit-tu-u . . . following a suggestion by Ylvisaker.

Rev. 2. The reading a-mu-a-ta is quite certain but the form $\mathrm{i}-\mathrm{mu}-\mathrm{a}-$ ta, "will he die," referring then to Ašurbânipal, instead of to the writer, suits the context of both prayers much better. Nowhere else is there a question concerning the writer's personal welfare. The motive of both texts seems to center in the king and his well-being in the present emergency.

Rev. 4. For similar ezib ša formulae in prayers of this kind, cf. Knudtzon, III $c$.

4. rev. 4, we would expect iz-za-az-zu-ni instead of iz-za-a-[zu-] $\mathrm{ni}$. The text, however, seems to exclude the latter reading.

4. rev. 5. On idakku-u, cf. Knudtzon, 57, rev. 4. We should expect the ending $\mathrm{n}$ i to be attached to both verbs of this line. The restored reading, however, follows traces which I felt excluded this in both cases.

\section{7. $83-1-18,45$}

OBVERSE

. . . ša šal Za-ku-u-te amat šam il Sin-a-[hê-rî̉ba]

[ummi ša] m Ašur-ah-iddina šar mât Ašur ki

3. [ittim il Ša $]$ maš-šum-ukîn ahi ta-li-me-šu

[šam il]Samaš-mîti-uballiṭ ù

[șih-ru]-te ahêe(pl)_šu itti zêr šarri itti

6. ...... pl amêl pihâti amêl ša-ak-ni

..... pl amêl âlik pâni itti amêl zak-ki-e

[amêl pi-kiit-]tu mâti gab-bu u itti mârê mât Ašur

9. [itti ka-]lu amêlu edlu sinništu man-nu ša ina libbi a-di-e

[ma-am-me]-te ša šal Za-ku-u-te amti ina mubhi

[m Ašur-bân]-apli mâr lib-lib-bi ša bidûti itti nišê mâti gab-bu

12. [la is-]sal-ú-u-ni man-nu ša a-bu-tu la di-iḳ-tu

[la ța]-ab-tu ù na-bal-kat-tu

[ina muhhi] $m$ Ašur-bân-apli šar mât Ašur bêli-ku-nu

15. [ta-as]-sa-li-a-ni te-ip-pa-ša-a-ni

.... la da-an-ku da-ba-a-bu

[la ța-]a-bu ina mubhi m Ašur-bân-apli šar mât Ašur

18. [bêli-ku-nu i]na libbi-ku-nu ta-nak-kir-a-nin-ni

.... a-a-ni us-su-uk-tu

[la di-iḳ-]tu mil-ku la ța-a-bu ša si-bi bar-ti 
21. [at-tu-]nu ina muhhi $m$ Ašur-bân-apli šar mât Ašur bêli-ku-nu [ta-mal-li]-ka-a-ni ta-dab-bu-ba-a-ni

. . . . . ûmu šani-e ina mubhbi du-a-ki

24. [m Ašur-bân-apla šar] mât Ašur bêli-ku-nu ta-dab-bu-ba-a-[ni]

........ il SAG.ME.GAR ilDIL.BAT

EDGE

$\ldots \ldots$... duti ... ûl ...

REVERSE

.... . u-te(?) . . . . .

[šum-ma] at-tu-nu TA.TA ûmê(me) an-ni-e

3. [. . . la] di-iḳ-tu ša si-hi bar-te

[ina muhbi] m Ašur-bân-apli šar mât Ašur be-lí-ku-nu

[ta-kab]-bu-u-ni la tal-la-ka-nin-ni

6. [uz-ni] ša šal Za-ku-u-te ummi-šu ù ša m Ašur-bân-apli

[šar mât Ašur] bêli-ku-nu la tu-pat-ta-a-ni ù šum-ma

[at-tu]-nu ša da-a-ki ù hul-lu-ḳi

9. [m Ašur]-bân-apla šar mât Ašur bêli-ku-nu ta-šami-ma-a-ni

[la tal]-la-ka-nin-ni uz-ni ša šal Za-ku-te ummi-šu(?)

[ù] ša $m$ Ašur-bân-apli šar mât Ašur be-lí-ku-nu

12. [la tu-pat]-ta-a-ni ù šum-ma at-tu-nu

[mim-ma] la da-an-ḳu ina [muhhi] $m$ Ašur-bân-apli

[šar mât Ašur bêli]-ku-nu i-nak-kir-an-ni

15. [ta-kab-ba-a-]ni la tal-la-ka-nin-ni

[ina pân šal Za-ku-]te ummi-šu ù ina pân $m$ Ašur-bân-apli

[at-tu]-nu la ta-kab-ba-a-ni

18. [šum-ma] at-tu-nu ta-šam-ma-a-ni

. . . . kal ṣâbê mu-šam-hi-iṣ-ṣu-u-te

[ra-bu]-u-te ina bir-tuk-ku-nu lu-u

21. .... ni lu-u ina amêl šakê lu-u ina ahêt(mêš)-šu

[lu]-u ahêt(mês)-ku-nu lu-u bêl ța-ba-te-ku-nu

[nišê] mâti gab-bu ta-šam-ma-a-ni

24. .... Š SL ta-ṣab-ba-ta-nin-ni

....... aš ... šal Za-ku-[te ... .

(Obv.) The compact of Zakutu, the maid of Sennacherib, the mother of Esarhaddon, king of Assyria, with Šamaššumukîn, the companion brother of Šamašmîtuballit and his younger brothers; with the seed royal and .... the governors, the prefects . . . . the deputies, with the magistrates, with the officials of the whole land, and with the Assyrian people, with every man and woman; 
whoever are averse to the agreement and oath of Zakutu the maid with the people of the whole land, concerning Ašurbânipal, the son of the offspring of favor; whosoever you are, who are parties to and who put into effect a wicked and treasonable plot and insurrection against Ašurbânipal, king of Assyria, your lord; with respect to the wicked plot, the treasonable plan against Ašurbânipal, king of Assyria your lord, you who in your hearts are hostile to me . . . . you who counsel and discuss a wicked scheme, a treasonable counsel concerning the uprising of a revolt against Ašurbânipal, king of Assyria, your lord, you who speak . . . . the second day, concerning the murder of Ašurbânipal, king of Assyria, your lord . . . . Jupiter, Venus .... (rev.) . . . . if you, from this day, shall utter a wicked speech, concerning the uprising of a revolt against Ašurbânipal, king of Assyria, your lord, if you do not come to me, if you do not reveal it to Zakûtu, his mother, and to Ašurbânipal, king of Assyria, your lord; and if you hear of a plan to murder and assassinate Ašurbânipal, king of Assyria, your lord, if you do not come to me, if you do not reveal it to Zakûtu, his mother, and to Ašurbânipal, king of Assyria, your lord; and if you utter anything unfavorable against Ašurbânipal, king of Assyria, your lord, which is hostile to me, if you do not come to me, if you do not speak before Zakûtu, his mother, and before Ašurbânipal, king of Assyria, your lord; and if you hear, whether you hear among any of the soldiers, the leading fighting men in your midst, or .... or among the captains, or among his brothers, or among your brothers, or among your friends, the people of the whole land, .... you seize me . . . Zakûtu

The compact by which Zakûtu, the grandmother of Ašurbânipal, bound the royal family, the aristocracy, the government officials, and the whole people not to revolt against Ašurbânipal, nor, in any way, to aid or abet disloyalty of any kind and, on the other hand, to report all such cases either to Ašurbânipal or his grandmother.

The date of this document lies between the accession of Ašurbânipal and the revolt of Šamaššumukîn. Ašurbânipal is repeatedly named as king and Šamašsumukîn stands first in the list of loyal subjects. Are we dealing then with a coronation document? The fact that the text makes Zakûtu alone initiate this compact and carry it out "in behalf of " Ašurbânipal favors this hypothesis. If Ašurbânipal had for some time exercised the sovereign authority, we should, at least, expect him to be a joint partner to such an 
executive proceeding as the above. In fact, any other occasion than his coronation makes Ašurbânipal too much of a weakling to be taken seriously; accordingly we may date the document in 668 B.c. The whole text is probably a single sentence constituting the oath of allegiance to the new sovereign, the apodosis being omitted or retained till the end, which has been lost.

Aside from the general character of this rather unusual text, three points demand special notice. First, the absolute supremacy assigned to Ašurbânipal, there being no hint here of any joint rulership of the two brothers. Ṡamaššumukîn's only distinction is to be the first of those who promise submission. Secondly, the queen mother of Esarhaddon was earlier known as $\mathrm{Ni-ik-a}$, cf. Knudtzon, 101, obv. 2, etc., but Johns, $A D D, 70$, a contract of the queen mother's sister, mentions the former as Zakûtu. Cf. John's remarks on 83-1-18, 45 in PSBA (1905), p. 94 (ibid., Laws, p. 370), based apparently on private notes, where he designates this text as a proclamation. Thirdly, the remarkable authority of Zakûtu, practically a queen regent for two generations, for in our text she not only promulgates the oath of allegiance to Ašurbânipal but, in the body of the agreement, she makes herself joint ruler to the extent of putting her own name first in regard to matters that may require executive action. Further, on the position of the queen mother in general and of the mother of Esarhaddon in particular, cf. Klauber, $24 \mathrm{f}$, Johns, LCL, 369 ff.; Winckler, AOF (1898-1900), 187-89. A subsidiary fact that heightens the effect of the authority of this woman is her earlier position as a mat, maid, concubine of Sennacherib, obv. 1, and the further fact that she apparently permitted this designation to cling to her even after she had become the first lady in the land, obv. 10.

Obv. 1. On the nominative form Zakûtu, cf. $A D D$, No. 645 (82-5-22, 90 ), on the basis of which Johns first identified Naika (Nakia) with $\mathrm{Za}$ kûtu ; cf. Meissner on Naki'a in MVAG, VIII, 12-15.

The exact word to be supplied may not be certain but a mât meets the requirements.

Obv. 2. [u mmi ša] is verified by rev. 6 and 16 .

Obv. 3. TA, i.e., [itti] is required at the beginning of the line by the context, the spacing, and the usage below.

Obv. 4. [ša . . at the beginning is required from the talîme-šu preceding. On Šamašmîtuballiț, cf. Behrens, $26^{1}$.

Obv. 5. . . . te might conceivably be the end of a proper name. If so it is certainly not the name of any of the sons of Esarhaddon so far known, nor would such a name meet the requirements of the context. What we clearly need is a term which will include all the brothers of Ašurbânipal. Šamaššumukîn the eldest, at least apart from Ašurbânipal, (cf. Godbey, $O S P, 42 \mathrm{ff}$., and Johns, $A J S L, \mathrm{XXII}, 232 \mathrm{f}$.) is appropriately named first, and [sibru-]te is therefore the most natural word to include all the rest. Zêr šarri will then include other royal kinsman of the reigning house, as well as of previous reigning houses, i.e., the seed royal. 
Obv. 6-7. Two officials are missing at the beginning of these lines. The singular forms are to be taken collectively. For the occurrence of these officials in the H. letters, cf. Godbey, OSP, 10-18, Klauber, 8.

Obv. 8. The word ending in . . . . tu is conditioned by the mâti gabbu following and for that reason it would hardly seem to be another class of officials. What is needed is a term to contrast with the mârê mât Ašur. I would read, therefore, as above, amêlpikittu, regarding the term as a summary of all the appointed officials.

Obv. 12. I take is-]sal-ú-u-ni as $I^{2}$ of לै- For di-ik-tu= $\mathrm{da}-\mathrm{mi} \mathrm{k}-\mathrm{tu}$, cf. Klauber, $43^{2}$, for discussion and references.

Obv. 16. The missing word is some such synonym of dabâbu as têmu, milku, or possibly dibbu.

Obv. 18. T]A at the beginning I regard as ina. For ta-nak-kir-anin-ni we may read ta-nak-kil-a-nin-ni, but in view of the $3 d$ pers. construction in rev. 14, I have accepted the former.

Obv. 19. Ussuktu I would regard as the feminine abstract from the same root as usukku, $H W B, 108 \mathrm{f}$., "eingeschlossener Raum," and so in the abstract, that which is shut off, secret, hidden, applicable then to secret plan, plot, which suits the present context.

Obv. 20. My text reads mil-lu; for the emendation mil-ku I am indebted to a suggestion from Dr. Ylvisaker.

Obv. 23. The lack of a clue to the first word destroys the context from here to the end of the obverse. du'aki inf. of Tרד, cf. Ylvisaker, § 32.

Rev. 1 may contain the name $\mathrm{Za-ku-te.} \mathrm{The} \mathrm{line} \mathrm{is} \mathrm{a} \mathrm{mark} \mathrm{of} \mathrm{sepa-}$ ration. What precedes consists of a series of indirect conditions; what follows presents a series of direct conditions, each introduced by $\mathrm{s} u \mathrm{~m}-\mathrm{ma}$, cf. rev. [2], 7, 12, and [18].

Rev. 2. TA=ištu; the second TA I would regard as a case of dittography. Or should we read ta-ta-tam-me? line.

Rev. 3. Amâta is perhaps the form lacking at the beginning of the

Rev. 7. tu-pat-ta-ni requires uz-ni.

Rev. 22. ta-ba-te- really ta-zu-te, in my text probably a scribal error. On bêl țâbti cf. Klauber, $A J S L$, XXVII, $331 \mathrm{ff}$.

10. Bu. $89-4-26,4$

OBVERSE

(Practically disintegrated.)

REVERSE

[m il] Marduk-šar-uṣur . . . . . .

[p]a-ki-id is-si-i ......

3. e-pu-uš u-ma-a . . . . . .

di(?)-e-ni la-a e-pa-aš

da-ba-bu ša šarri la iš-me 
6. e-ni ša amêl pibâti i-da-gal

a-ni-na šarru be-lu di-e-nu

šá abi-ka e-pu-šu-u-ni

9. tee-e-mu iš-ku-nu-u-ni

ú-ma-à an-nu ú-sa-bal-ki-tu

ù a-na-ku itti bît abi-ia gab-bu

12. ki-i kal-bi a-sa-ap-pu-'u

ana me-ni-ia la ab-ri-id

ma-șar-tu ša šarri bêl-ia la-ṣur

15. dûru ma-ki-i šarru šarru be-li

ki-i šá i-la-u-ni li-pu-uš

man-nu at-ta amêl dupšarru

18. šá ta-sa-su-u-ni

ištu pân šarri bêli-ka la tu-pa-zar(?)

ța-ab-ti ina pân šarri ki-bi

21. Bêl il Nabû ța-ab-ta-ka

ina pân šarri like-bi-ú

(Rev.) Mardukšaruṣur . . . . the officer . . . . has done as follows . . . my rights he does not establish. He has not hearkened to the word of the king. He is subservient to the governor, since the time the king, my lord, began to render to me the justice of thy father and to place it on record. Now they stir up opposition; and I, with the whole house of my father, am supplicating like a dog. Why am I discarded? I would keep the watch of the king. The fortress is neglected(?). May the king my lord act according to his pleasure. Whoever thou art, O secretary, who dost read (this) before the king thy lord, do not conceal my good deeds. Declare them before the king. May Bêl and Nabû declare thy good deeds before the king.

The appeal of a former favorite of royalty for deliverance from the oppression of subordinate officials and for restoration to a place of trust. The obverse apparently recounted the writer's past faithfulness, cf. rev. 19-20.

Rev. 1. Owing to the incomplete lines, the relation of Mardukšarusur to the writer is not clear. For a man of this name, ef. Klauber, 75; Johns, $L C L, 352$.

Rev. 7. a-ni-na, as suggested by Ylvisaker, is to be taken as equivalent to eninna.

Rev. 12. On a-sa-ap-pu-u from sapû, pray, supplicate, cf. H. 382, obv. $6 ; 659$, rev. 8 , and Ylvisaker, 53. 
Rev. 13. Is ana(TA) me-ni-ia to be taken as an equivalent to a mmêni? The ground meaning of harâdu is uncertain; cf. $H W B$ and Behrens, 8 .

Rev. 14. la-șur for lu-aṣṣur, prec. of צבצר.

Rev. 15. ma-ki-i, I would regard as a pm. of -12:2; cf. $H W B, 407 a$, and Winckler, Amarna Briefe, 16:18, im-te-ki, "neglect" (?).

\section{1. $83-1-18,53$}

\section{OBVERSE}

. . . . . . a-ni-ni ša ina ḳabal-tim

. . . . . . amês Pu-ḳu]-du ina muh-hri-ni

[ana muhhhi šarri] bêl-i-ni ni-sap-par-ma

. . . . e e]-mu-ḳu a-na ki-tir-i-ni

6. [li-is-sa]p-pa-ra u amêl Gu-ra-sim-mu

. . . . t]i-ni ú-šu-uz-zu amêl nakri a-na muh-ḩi-šu-nu

. . . . i]l-li-ku aš-šu i-mu-ru-ú-ma

9. țe-e-mu ša mât Ašur ki la-pa-ni-šu-nu i-ri-ḳu

u man-ma amêl bêl pibâti a-na ki-tir-šu-nu

la il-li-ku ḳâtâ a-na amêl nakri it-tan-nu

12. NUN.KI u Uruki ša ri-e-hi ki-i

il-'i-u it-ti amêl nakri it-ta-ši-iz-zu

a-du-ú amêlGu-ra-sim-mu gab-bi it-ti-ik-ru

15. alu ina lib-bi ša it-ti mât Ašurki ú-šu-uz-zu

ia-a-nu al-la Uruki al Ki-sig

$u$ al ŠA-AD. iddina $u$ šarru bêl-a-ni i-di

18. ki-i Uruki ina ḳabal mât Akkadî ki i- . . . .

\section{EDGE}

šú-u

\section{REVERSE}

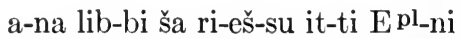

ḳa-ta-a-ni amêl Pu-ḳu-du u mât Tam-tim

3. i-zi-'i-ru-na-a-šu u lum-nu a-na bît ilâni(pl)-ka

kap-du ina da-a-ku u bu-bu-ta-a-nu

uk-ta-at-tu-na-a-šu kul-la-a-ti

6. ma-ku-tu ina kâti-šsu-nu ni-it-tal-lak

a-du-u mât Tam-tim mât $\mathrm{Pu}$-ḳu-du u amêl Gu-ra-sim-mu

di-ki a-na muh-bui-ni it-ta-šú-ú-ni

9. šarru bêl-a-ni e-mu-ḳu a-na ki-tir ša bît ilâni(pl)-šu

[liš]-pur nikasi ša šarrâni abê-ka a-na il Sin

[id]-di-nu ḳâti amêl nakru-tim ta-kaš-šad u mâti ina ḳât šarri

12. [i-te]-el-lu-ú u mât Ašurki ina pa-ni-šu-nu

. . . . amêl rabûti ša šarri a-na ma-aṣ-ṣar-ti

i-ni it-ta-aš-šu i-ši-ru-u . . . . . . . 
15. ka-ad hu(?) bu(?) . . . . . .

........ šarru . . . . ni

$\ldots \ldots \ldots \ldots$

.... W]e who in the midst of . . . the P]ukudu against us. [Unto the king] our lord, we have sent and . . . . troops for our assistance [let him s]end. The Gurasimmu . . . . have been loyal, the enemy against them . . . . has come, because they have seen this and because news from Assyria is lacking and none of the prefects has come to their assistance, they have given their support to the enemy. Eridu and Ur, which are left, have chosen to go over to the enemy. Now all the Gurasimmu have become hostile. There is not a city among them which has remained loyal to Assyria more than Ur and Kisig and ŠA.AD. iddin-na and the king, our lord, knows that $\mathrm{Ur}$ is in the midst of Akkad . . . (Rev.) because of its preeminence(?) with its temples we are ruined. The Pukudu and the Sealand hate us and they plan evil against thy temples. With slaughter and raids they are making an end of us, everything is destroyed, we shall fall into their hands. Now the Sealand, the Pukudu, and the Gurasimmu are mustering, they have risen against us. Let the king, our lord, send troops for the protection of his temples. The treasure which the kings, thy fathers, gave to Sin, the enemy will capture, and the land will slip away from the hand of the king, and the land of Assyria before them . . . . The officials of the king lift up their eyes toward the fortress. . . . .

This letter consists of an appeal for reinforcements and furnishes a glimpse into the situation in lower Babylonia during the earlier stages of Ṡamaššumukîn's revolt, ef. No. 12. Although the introduction is lacking, it seems evident from the first plural throughout, together with the cities named, that it is a community which addresses the king; and from the prominence given to Ur and its temples, in particular, to the temple of the god Sin, one might refer our text to the people of Ur. We have also letters from Kisig, ef. H. 210, 736, 1121. Furthermore a comparison of the present text with H. 942 may point to the people of the city of Šrtena(?) as the authors. For the reading of the name, cf. Bezold, Catalogue, V, p. 2183, and compare the writing of the name in H. 942, obv. 11, a' ${ }^{\mathrm{S}} \mathrm{A}$. NIN. iddin$\mathrm{n}$ a with the present text, obv. 17, SA.AD.iddin-na. I am inclined to think that we have the same name underlying these three forms, perhaps more abbreviated in one case. A fresh collation of the tablets may determine the matter. 

32-34.

Obv. 3. On the occurrence of the Pukudu in H. cf. Streck, $M V A G$, IX,

Obv. 6. On the reading Gurasimmu ef. Streck, $A J S L$, XXII, 212, but see Schiffer, 123, where the present reading seems to have been overlooked. Some form of is to be restored in this line, perhaps the $I^{2}$ precative as I have indicated.

Obv. 12. rêhi pm. of ריז.

Obv. 13. The last sign of this line in my text is SÍB, i.e., rê' $\hat{u}$. Dr. Ylvisaker's suggestion to separate it into iz-zu commends itself by the context thus secured, while the change involved is very slight.

Obv. 16. On ianu, cf. Ylvisaker, $\S 34$.

Obv. 17. For the rendering of alla see Ylvisaker, 52 f.; but cf. Thompson, $L B L$, p. xxxvi, and glossary, p. 199.

Rev. 1. rešsu lit. its head, i.e., perhaps "its headship"(?). E pl-ni may be an abbreviation for bît ilâni, ef. rev. 3 and 9 .

Rev. 8. My text reads id-di-šú-ú-ni. Perhaps it is better to read it-ta-šú-ú-ni, ef. H. 942, rev.6.

\section{K. 471}

\section{OBVERSE}

a-na šar mâtâti bêl-i-ni ardâni-ka

amêl Urupl amêl ši-bu-tu

3. $u$ amêl șihrûte ${ }^{\text {il }}$ Sin $u$ il NIN.GAL

a-na šar mâtâti bêl-i-ni lik-ru-bu

umu-us-su ${ }^{i 1}$ Sin $u$ il NIN.GAL.

6. a-na balât napšâti ša šarri bêl-i-ni nu-ṣal-la

a-ni-ni u( ?) $m$ il Sin-tab-ni-ușur

ma-aṣ-ṣar-ti ša šarri bêl-i-ni

9. ni-it-ta-șar a-na bu-bu-ti-ía

[šî]ra ša amêl mârê (pl)-ni u mârâti (pl)-ni

[tul-t]a-kal-u-ni ma-aș-ṣar-ti

12. [ša šarri] bêl-i-ni ûl nu-maš-šir

[m il Sin]-tab-ni-uṣur ša e-ku-ti

[u bu-bu]-ti ina ma-aș-șar-ti

15. [ša šarri] bêl-i-ni it-ti-ni

[u-ša-a]m-kir-u ina šêpâ

[m il Šam]aš-šum-ukîn iṣ-bat

18. . . . . . aš-šu a-di . . .

$\ldots \ldots$. ḳa aš-šu a-di

. . . . . . il-li-ka 
. . . Š̆amaš-šum-uk[în . . .

ni(?)-iš êni a-na pa-an

3. šarri bêl-i-ni ûl il-li-kan

en-na ki-i il-li-ka

dib-bi ša iḳ-bi(?)-ma a-ta(?)

6. u sal-lu la-a ištu ša ram-ni

a-na mub-bi m il Sin-tab-ni-uṣur

arad amêl da(?)'a-an-nu

9. ša šarru bêl-i-ni i-dib-bu-ub

šarru bêl-a-ni lu-ú i-di

(Obv.) To the king of the lands our lord. Thy servants, the people of the city of Ur, old and young. May Sin and NINGAL be gracious to the king of the lands, our lord. Daily unto Sin and NINGAL do we pray for the life of the king, our lord. We and Sin-tabni-uşur have kept the watch of the king, our lord. For my hunger wilt thou make me to eat the flesh of our sons and daughters? The watch of the king our lord we have not forsaken. Sin-tabni-uşur, whom distress and hunger, in the watch of the king our lord with us, have caused to revolt, has submitted to Šamaššumukîn [three lines too broken to translate]. (Rev.) . . . . Šamaššumukîn . . . . the lifting up of the eyes, before the king, our lord, he did not come. Now that he has come, the word which he has spoken ........ captured(?) not because of myself. Concerning Sin-tabni-usur, the servant of the judge of whom the king our lord has spoken, the king, our lord, will surely know.

In this letter we have both an appeal and an apology from the citizens of "Ur of the Chaldees," presenting the counterpart to the situation in No. 11 , probably at a somewhat later stage of the revolt. While the outlook in No. 11 is undoubtedly desperate enough, cf. rev. 2-13, still the conditions are more general, other cities are included, and there is no reference to the straits of famine nor is there evidence that overtures have already been made to the rebels, such as characterize the present text. This letter when complete may have thrown some valuable light on the question whether Sin-tabni-uṣur, the governor of Ur, yielding to the pressure of Šamaššumukîn, actually revolted, cf. Johnston, JAOS, XVIII, 148-49; Johns, LCL, p. 354. Unfortunately the text is too badly damaged at the very points where the desired information might have been forthcoming. The restorations proposed, particularly in obv. 16 , if assured, would confirm the contention that 
Sin-tabni-usur under pressure of siege and famine finally took the side of the rebellious brother.

Obv. 7. On Sin-tabni-usurur's governorship and his relations to the king ef. Johnston on K. 824, JAOS, XVIII, 148-50, and Klauber, 101 f., on H. 754.

Obv. 11. The conditions of the line suit the reading tultakaluni

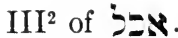

Obv. 16. The traces before kir may stand for a m. 


\section{TEXTUAL NOTES ON THE LETTERS OF THE SARGON PERIOD}

The following textual comments attempt to cover the chief points where Harper's Texts have thus far been brought in question and consist of a comparison of the variant readings with the originals in the British Museum. Different readings in portions restored have been omitted, as these involve no comparison with the originals. For convenience the order followed is that of Harper's numbering.

H. 5 (K. 583, Obv. 15) Delitzsch BA I 627 reads . . . . ili for in a pa-an, but compare Obv. 15 , note 6 , which recognizes an omitted sign before . . . . ili, "das am meisten mit GUR Ähnlichkeit hat." The form in question seems to consist pretty plainly of one sign written over another (1). ${ }^{1} \quad$ I would read with $\mathrm{H}$. in a pa-an.

H. 6 (K. 595, Obv. 7) van Gelderen BA IV 505 transliterates da-ab-b[a]-ni. The traces in H. permit this, but the tablet has one more horizontal stroke (2) than in $\mathrm{H}$., which makes the reading ba impossible; Rev. 11 van Gelderen $B A$ IV 503 reads us-sa-bi[1]. Behrens $A B B 91$ questions what sign is to be restored, whether rik, li, or some other. The difficulty is due to a typographical error. A perpendicular wedge has been omitted in $\mathrm{H}$. or has been drawn in the process of printing. The sign (3) is quite surely rik. Rev. 14 Behrens $A B B 91$ corrects pi to ši and reads a-du ištên li'mi-šu for a-du diš-pi-šu. The form consists of one sign written over another, but whether it is composed of ši written over pi or vice versa, it is impossible to determine from the tablet. The context must decide.

H. 23 (K. 602, Obv. 15). The tablet has been cleaned since first published, and two strokes in addition to the traces given in $\mathrm{H}$. can be seen (4). Compare Behrens' assertion, $A B B$ 98, that the traces

${ }^{1}$ Figures in parentheses refer to cuneiform notes at the end of this paper. 
point to tarbaṣu. The traces do lend themselves to tarbaṣu. This line extends three millimeters beyond the beginning of the previous line and two millimeters beyond the beginning of the line following. Obv. 16 Zimmern $A B B 98$ reads zi-in-na-tu for nam-in-na-tu with Bezold $Z A$ II 446 . This typographical error is probably due to the nam just above. The correction is to be accepted.

H. 29 (K. 1204, Obv. 9) Behrens $A B B 34$ proposes an or nu for maš in ḳur-ba-maš. It is better to read an instead of bar, maš. The sign as it stands can be taken in either way.

H. 36 (K. 1032, Obv. 7) Behrens $A B B 29$ queries whether the fifth sign (uncertain) may not be ra and reads: ${ }^{1}$ Har-ra-an. The traces plainly do not lend themselves to $\mathrm{ra}$, but rather to $\mathrm{ur}$. The text in $\mathrm{H}$. lacks one perpendicular at the right as well as an additional horizontal wedge beyond (5). Rev. 6 Behrens $A B B 29$ remarks: "Die Schreibung ist sehr absonderlich." There seems to be no doubt, however, as to the correctness of the text in $\mathrm{H}$. Rev. 9 Behrens $A B B 29$ is inclined to read bēl a-[gi-e] or barran for the third and fourth sign. The second sign, EN in $\mathrm{H}$., is badly rubbed. It seems, however, to be quite certainly Eš (sin), followed by traces of a sign erased. The third sign is a scribal, not typographical error for $\mathrm{EN}=\mathrm{b} \hat{\mathrm{l}} \mathrm{l}$. There is also beyond $a$ some very badly rubbed, though fairly certain traces. For the entire line cf. (6). Rev. 11 Behrens $A B B 29$ reads ni-ip-bi for ni-ur-bi. This typographical correction is to be accepted.

H. 43 (K. 122, Rev. 10) van Gelderen $B A$ IV 516 (note) reads KU-U-ŠAG for KU-šAG. This correction is to be accepted. Cf. Rev. 16 and 21. Rev. 17 van Gelderen $B A$ IV 514 reads wa-lu-ub for pi-lu-ub, but the text requires gal-lu-ub, already noted by Harper AJSL X 200, "At present I am inclined to regard the pi as an incorrect reading of gal. It will be necessary to consult the original of this difficult text again."

H. 47 (K. 979, Obv. 8) Harper AJSL X 196 reads ri-in-ku for $\mathrm{ri-LUGAL}-\mathrm{ku}$. This correction is to be accepted.

H. 52 (K. 80, Rev. 1) Streck $A J S L$ XXII 218, note 29, calls attention to S. A. Smith's reading sa-si-ka-na for sa-si-ka-ni in H. The sign in question is to be read na with Smith, although the 
form is peculiar. Rev. 10: tu-ša-lik. According to H. (footnote) lik is written over ba. Behrens $A B B 51$ questions which is superimposed. Either one is possible. It is a very deeply cut, clear writing of both signs.

H. 56 (K. 649, Obv. 7) Behrens $A B B 46$ reads ma-ak-lu for is a $\mathrm{k}-\mathrm{lu}$ in H.; is, however, stands in the text and corresponds to is in Rev. 5; m a seems impossible, the third upper horizontal stroke would have been too high. The sign is deeply and plainly cut.

H. 86 (K. 660, Rev. 5) Johnston JAOS XVIII 169 transliterates re[šni], and remarks that the traces indicate si, but might be iš, and that $\mathrm{ni}$ is omitted because of the following ni. I would read provisionally ri-e-šu (7); ri is written over an erasure, e is broken, and šu rubbed and broken.

H. 89 (K. 515, Rev. 5) Johnston JAOS XVIII 173 reads tupi-iš for tu-ši-iš, followed by Behrens $A B B$ 54. I would read pi with Johnston. It seems quite certain, however, that the scribe first wrote ši, which he then altered to pi. It differs very much from pi in Rev. 7 and Obv. 12. Harper had already suggested the above correction; ef. AJSL X 199, "My text reads ši, but I am inclined to think that this character is pi."

H. 94 (K. 1147+1947, Rev. 1) Streck AJSL.XXII 220, note 41, transliterates Ru-ka-ba-a ša and emends ša to a. According to the footnote in H. the last sign of Rev. 1 is perhaps a. The text (8) is clearly as given in H., which is plainly meant for a, as this letter is in the Assyrian script.

H. 97 (K. 4770, Obv. 7) Behrens $A B B 46$ reads i-su-ri for $\mathrm{i}-\mathrm{su}-\mathrm{bu}$. Behrens' correction of this typographical error is to be accepted.

H. 110 (K. 576, Rev. 6) Behrens $A B B 17$ reads li-bi me-e ${ }^{\mathrm{pl}}$ for li-kaš-šip mê. I would read with $H$. a ${ }^{p l}$. The $m \hat{e}$ has been rubbed, but I cannot see any cut strokes before the a.

H. 113 (K. 501, Obv. 13) Behrens $A B B 40$ reads ma-șar for is , plus a broken sign. This correction, already made by Harper, $A J S L \mathrm{XV} 142$ is to be accepted. I am sorry to be compelled to state that Behrens, in many places, either does not know the literature on the Letters, or fails to give credit to others whose statements he has accepted 
H. 114 (K. 538, Rev. 10) Behrens $A B B 85$ remarks on ta-buu-ni: "Die Endung - uni . . . . ist sehr auffällig." This reading is also plainly and unmistakably correct.

H. 137 (K. 467, Obv. 10) Jastrow RBA XIV 518 reads lum-n u for lum-kur. This correction is to be accepted.

H. 138 (K. 469, Rev. 13) Johnston JAOS XVIII 169 reads u-qa-ib-u(?)-ni for u-qa-ib-ni, and remarks "The enclitic ni cannot be joined to the verb without a union vowel (Del. Assyr. Gram. p. $79 \beta) . .$. The insertion of $u$ improves both the sense and the construction." The text in $\mathrm{H}$. is correct, there is no $\mathrm{u}$.

H. 141 (K. 481, Rev. 3) Jastrow RBA XIV 532 reads "fourteen" for "four" and remarks, "Bei H. fehlt der Winkelhaken." The text is correct. One can see traces of $u$ through which erasure lines have been drawn. These extend into the ud preceding (9).

H. 149 (K. 540, Obv. 8) Meissner $S A W 46 a$ reads (ku(!) zip-pi for ki-zip-pi, followed by Behrens $A B B 33$. The sign is correct in $\mathrm{H}$. and accurately reproduced. It corresponds exactly to the sign after Akkad Obv. 9, which of course must be ki.

H. 167 (K. 582, Rev. 19) Behrens $A B B 10$ remarks concerning ut-ru, "Es hat beinahe den Anschein als ob statt ut vielmehr dà (kal) zu lesen sei." The text in H. is undoubtedly correct.

H 201 (K. 690, Rev. 5) Zimmern's suggestion in $A B B$ 31, that ila'uni may have stood after bêli-ia is to be accepted. I have had the tablet cleaned and one can now see ša šarru be-li i-la$[\mathrm{u}-] \mathrm{ni}$. The $\mathrm{ni}$ is broken and rubbed; ia does not stand in the text; cf. Obv. 4.

H. 281 (K. 13, Obv. 30) van Gelderen $B A$ IV 528 deliberately leaves out the first sign, ri, in his translation. Johnston $J A O S$ XVIII 141 reads ta (?). I cannot accept the reading ta. Nevertheless a sign is undoubtedly to be read though it is on a corner edge and badly damaged. However, it does not seem to me that the traces lend themselves to $\mathrm{ri}$ of $\mathrm{H}$. and of Pinches (cf. IV, R. 52, No. 2), but rather to an imperfectly cut la (10). It is not in the scope of these notes to go beyond textual matters. I would, however, only call attention to the effect of la upon the translation in giving force and clearness to the speech of Ummanaldaš. For 
translations cf. Johnston JAOS XVIII $139-40$ and van Gelderen $B A$ IV 528-29.

H. 328 (K. 638, Obv. 12) Delattre PSBA XXIII 52 reads amêl Kal-di for amêl Kal-ki. Delattre's correction of this typographical error is to be accepted.

H. 344 (83-1-18, 28, Rev. 1) Klauber $A B 53$ reads, ba-ri-ki-ili for ba-ri-di-ili. This correction is to be accepted.

H. 346 (Bu. 89-4-26, 9, Obv. 3) Streck AJSL XXII 214 reads ${ }^{\text {al }} \mathrm{kak}-\mathrm{zi}$ for al kak-nam. Streck's correction of this typographical error is to be accepted.

H. 353 (82-5-22, 169, Obv. 5) Behrens $A B B 74$ remarks that the ideogram for aribu is given more correctly by Bezold, Cat., p. 1841, than in H. Bezold's reading is typographically better than in $\mathrm{H}$.

H. 356 (Bu. 89-4-26, 160, Obv. 20) Behrens' remark, $A B B 71$ "dagegen sind die Spuren die auf nu deuten wohl falsch" is to be accepted. On the other hand, his endeavor to read ri instead is questionable. The traces are on a corner edge and badly rubbed, but there are more than are given by Bezold, Cat., p. 1929, or in $\mathrm{H}$. It is difficult to tell whether or not there is an upright wedge-head at the juncture of the lines at the right (11).

H. 358 (Rm. 76, Obv. 6) van Gelderen BA IV 510 regards ilu Ninib as a dittography. Jastrow $R B A$ XIV 540 remarks, "Harper hat ein zweites Ninib copiert." The text in H. is correct. Obv. 8 van Gelderen $B A$ IV 510 reads a [ši-]bu-te and regards ši as omitted by mistake. The text is correct. The error is scribal. Obv. 17 van Gelderen $B A$ IV 510 reads, i ḳ-ḳa-ka for iḳ-nak(?)ka. The sign interrogated is badly scratched, but nak(?) cannot be $\mathrm{ka}$. The top stroke of $\mathrm{nak}$ is very plain, as also are the two perpendicular wedges inside. $\mathrm{Nak}$ alone satisfies the writing. Obv. 24, e-me (cf. van Gelderen's note, $B A$ IV 511), is now correctly read nišê by Harper; cf. Klauber AJSL XXVII $332^{10 a}$. Rev. 9 van Gelderen $B A$ IV 511 points out a dittography in bêli. The text is correct. The error is scribal. Rev. 12 van Gelderen $B A$ IV 511 reads ina libbi ša-da-a u-kan-ni-šu-u-ni for ina libbi ša ša-a-u i-ni-šu-u-ni. The correction is to be accepted. Rev. 13 van Gelderen BA IV 511 reads ia for $z a$. The thought 
undoubtedly requires ia, but $\mathrm{za}$ gives the exact form on the tablet. If the scribe ever added a third upper stroke on the right it has been so completely effaced as to leave no incision whatever; ef. H. 964, Rev. 7, footnote. Rev. 14 van Gelderen BA IV 509 supplies mu and reads te $[\mathrm{mu}]$. The text is correct. The error is scribal. Rev. 15 van Gelderen $B A$ IV 511 adds [ni] to iš-pur-an. The text in $\mathrm{H}$. is correct. There is no trace of $\mathrm{ni}$, after an, on the tablet. Rev. 16 van Gelderen $B A$ IV 512 reads ia for $n i($ ?) in $\mathrm{H}$. The traces favor ia (12).

H. 366 (82-5-22, 96, Rev. 2) Behrens $A B B 46$ reads i-paaš-šar for i-pa-pa-šar. 'Behrens' correction of this typographical error is to be accepted.

H. 378 (83-1-18, 11, Rev. 5) Behrens $A B B 99$ remarks: "Ob pittit zu lesen, ist sehr fraglich." The text is correctly reproduced in H. (13). Cf. H. 917, Rev. 12.

H. 380 (Rm. 2, 3, Obv. 7) van Gelderen BA IV 522 reads $\mathrm{u}-\mathrm{a}$ for ellu. The portion taken as $\mathrm{u}$ is very closely joined to what follows (14), and on account of the spacing lends itself better to the reading in $\mathrm{H}$. The reading can only be determined by the context.

H. 399 (67-4-2, 1, Obv. 7) Delitzsch BA I 236 correctly reads ša taš-pu-ra. The line is omitted in $\mathrm{H}$.

H. 401 (83-1-18, 30, Obv. 10) Behrens $A B B 72$ reads pa-an. for maš-an. The lower wedge is indistinct and looks like an erasure. I would, however, read pa from the context and regard it as a scribal error. Obv. 10, the first sign is plainly correct and the interrogation mark is to be deleted.

H. 407 (83-1-18, 40, Rev. 1) Jastrow $R B A$ XIV 518 reads lum-nu for mi-nu. The traces in the text (15) may be taken in either way, to be determined only by the context.

H. 408 (Rm. 2, 1, Rev. 11) van Gelderen BA IV 542 reads liš for ši. As the line stands (16) ši is plainly in the text. But inasmuch as there are several erasures in the line and especially since the scribe utilized part of an erasure in writing $\mathrm{mu}$ (cf. note), so in the following sign, the horizontal wedge of ši, which is quite plain and shows little or no signs of erasure, was probably intended to be erased. The line, however, is to be read a țe-mu liš-kun. 
H. 409 (Rm. 2, 2, Obv. 3). The text is broken. The line is incomplete and should have been so indicated in $\mathrm{H}$. Cf. Klauber's note, $A B, 67$.

H. 414 (Rm. 77, Obv. 11) van Gelderen BA IV 526 reads ki-it-ki-te-e for ki-it-ku(?)-te-e. The text is badly rubbed, but what can be seen (17) seems to favor ku rather than ki, especially in view of the writings of $\mathrm{ku}$ on this tablet.

H. $426(80-7-19,17$, Obv. 11) Strassmaier $A V$ pp. 811, 978 reads li-iḳ-ḳa for li-ik-liš in $\mathrm{H}$. Johnston $A J S L$ XVII 150 reads with Strassmaier. I would accept Strassmaier-Johnston's reading. The sign is written over an erasure (18). Cf. H., footnote.

H. 438 (K. 177, Obv. 7, and Rev. 4) Zimmern $A B B 7$ remarks on il ši-da-da: "Dafür wird im Orig. wohl il ši-sig-sIG (Zeichen arqu) stehen." I cannot accept Zimmern's correction. The text is plainly correct.

H. 444 (K. 645, Obv. 13) van Gelderen BA IV 524 questions whether ali in $\mathrm{H}$. should not rather be lib. The traces in $\mathrm{H}$. are correct, whatever the reading. Obv. 13 Johns AJSL XXII 229 reads kap-pa-ri for bel ut-ri. I have had the tablet cleaned, but I can only read bêl as in $\mathrm{H}$. It is true the two wedges at the foot are both written inside the perpendiculars (19), which naturally favors kap, but there seems to be no room above for a third wedgehead. Also bêl in Obv. 3 is written in the same manner.

H. 450 (K. 898, Rev. 4) la-ku šu-tu-u-ni. The text is correct. ' Cf. Behrens $A B B 85$.

H. 458 (K. 1122, Obv. 8) Behrens $A B B 53$ reads man-daat-ti for u-da-at-ti. In my copy of $\mathrm{H}$. the first sign of Obv. 8 has been pulled out altogether. Of course man is to be read, which is plainly on the original. For other examples of press pullings ef. H. 222 (K. 186, Obv. 8), in a lib-bi. lib lacks the three perpendiculars; also in Obv. $11 \mathrm{ma}$ of $\mathrm{ma}-\mathrm{a}$ has been pulled.

H. 464 (K. 1519, Obv. 19) Behrens $A B B 3$ reads iḳ-bu-nu for a $-\mathrm{bu}-\mathrm{nu}$. The text in $\mathrm{H}$. is correct.

H. 473 (81-2-4, 65, Rev. 6) Klauber $A B 21$ reads be, bad for $\mathrm{bab}$. I am inclined to read with $\mathrm{H}$. This sign differs from the be below Rev. 19, in that the horizontal stroke is prolonged in line 6 . I would not regard the reading be as excluded. 
H. 493 (83-1-18, 13, Rev. 27) Behrens $A B B 65$ questions the reading ak-kud-di-ni. The text in $\mathrm{H}$. is correct.

H. 496 (K. 474, Rev. 6) Behrens $A B B 68$ reads tim-ma for in a ud-ma. The correction is to be accepted (20).

H. 506 (K. 678, Obv. 16, Rev. 5) Klauber $A B 9$ reads gab-bu for $\mathrm{mu}-\mathrm{bu}$. This typographical correction is to be accepted.

H. 514 (K. 477, Rev. 12) Behrens $A B B 30$ reads du-ul-lu for ilu ul-lu. The text is broken, but the reading $d u(21)$ is to be accepted.

H. 516 (81-7-27, 31, Obv. 11) Streck AJSL XXII 215 proposes to read the first sign us. The form on the tablet is correctly reproduced in $\mathrm{H}$. and, though unsatisfactory, it cannot be uš.

H. 517 (82-5-22, 91, Obv. 11) Zimmern $A B 13$ reads ța-ba-át for $\mathrm{da}-\mathrm{ba-bu}$ in $\mathrm{H}$. I have had the tablet cleaned, and I would read tu (22) rather than with either $\mathrm{H}$. or $\mathrm{Z}$. The sign runs over into šarru of Rev. 6 .

H. 518 (83-1-18, 27, Obv. 11) Behrens $A B B 19$ reads bul[u-pu], and remarks: "aber zu l[u] stimmen die Spuren bei Harper keinesfalls." The traces stand plainly as in H., hence the restoration $l u$ is impossible.

H. 520 (K. 680, Obv. 7 and 11) Streck $A J S L$ XXII 216 reads ak for Toffteen's ilu ak. The sign in $\mathrm{H}$. is neither one (23), but a typographical error for a $k$, i.e., the first perpendicular wedge should be deleted.

H. 552 (K. 640, Rev. 6) Toffteen $A J S L$ XXI 89 reads Ba-har-ri. Johns $A J S L$ XXII 229 reads correctly, with the text, Ba-kar-ri:

H. 553 (K. 659, Rev. 9) Behrens $A B B 100$ reads II šu for III šu. This correction is probably to be accepted. The text is badly damaged; only the lower ends of two wedges are visible. From the spacing, however, one could read III as well as II if the context demanded it.

H. 576 (K. 1009, Obv. 8) Klauber $A B 69$ reads te for tu, but the text is correct. $\mathrm{Tu}$ stands plainly on the tablet, although with Klauber one would expect te.

H. 649 (81-2-4, 110, Rev. 5) Johns AJSL XXII 231 reads 
correctly š u-b u-pa for Toffteen's misreading šu-bु u-ra (cf. AJSL XXI 99). The text in $\mathrm{H}$. is correct.

H. 652 (80-7-19, 22, Obv. 7) Behrens $A B B 25$ remarks: "Vor dem Personendeterminativ fehlt wohl nichts (?)" Ašur is written directly under pur above. Hence there is space for more than the broken determinative (24).

H. 685 (81-2-4, 96, Rev. 5) Streck AJSL XXII 208 remarks: "Vor ak-ta-la bietet $\mathrm{H}$. noch einen schrägen Keil; sollte letzterer ungenau für AS̆ stehen, mithin die Stadt Aššur (AŠ) gemeint sein?" This sign can only be a division-mark.

H. $688(80-7-19,21$, Obv. 8) Behrens $A B B \quad 78$ reads: BE-GIŠ-BI for BE-GIŠ-RIG(?). The text in H. is plainly correct and the interrogation is to be deleted.

H. 733 (81-2-4, 113, Rev. 9) Klauber $A B 31$ reads maṣar-tu for maşar ali. This correction is to be accepted.

H. 755 (83-1-18, 122, Obv. 6 and 16) Klauber $A B$ 73, note 3, compares the variant reading in Godbey's list, AJSL XXI 81. The text in $\mathrm{H}$. in both cases is correct, and Godbey is to be corrected accordingly.

H. 767 (83-1-18, 75, Obv. 9) Klauber $A B 7$ reads er meš for bi. The text in $\mathrm{H}$. is correct. The erasure is complete, the bi is very plain and deeply cut.

H. 789 (K. 1964, Obv. 7) Streck AJSL XXII 221 reads šal-lu-ki-e-a for šal-lu-ki-si-a. Streck's 'correction is to be accepted.

H. 802 (81-7-27, 39, Obv. 6) Streck AJSL XXII 217 suggests a $\mathrm{m}$ for the fourth sign. The text is correctly reproduced in $\mathrm{H}$.

H. 858 (K. 822, Obv. 16) Behrens $A B B 54$ corrects ši, lim to pi, and reads ta-bap-pi for ta-kal-lim; cf. Harper $A J S L$ XIX 231. The correction of Behrens is to be accepted.

H. 869 (81-2-4, 120, Obv. 6) Jastrow Babyloniaca III 3 p. 227 remarks upon "the gloss which Harper's copy, however, does not indicate as such." (1) The text in H. is given exactly as in the original; (2) $\mathrm{H}$. has reproduced the indenture which in this case shows the gloss; (3) the script is identical with that of the rest of the tablet; (4) it is not smaller, nor is it written above or below the word to be explained, as is usual in most glosses. 
1) Dar

4)
2) Arsts

3)

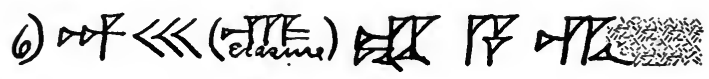

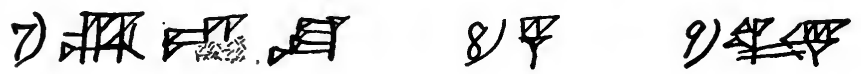

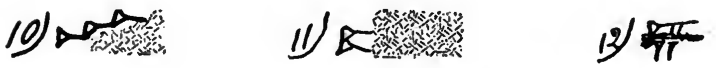

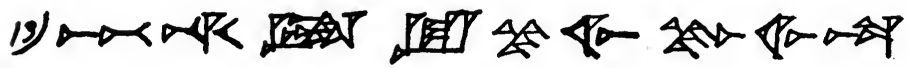

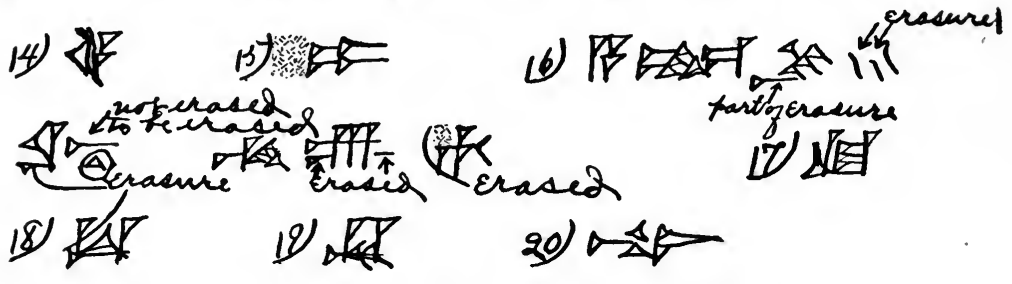

21) 22) 造点 23)

24) Perhafe [T

LoNDON

September 11, 1911

This article was in type before the appearance of Dr. Ylvisaker's excellent monograph, Zur babylonischen und assyrischen Grammatik, and hence no notice could be taken of the readings given by him on pp. 75-84.-R. F. H. 



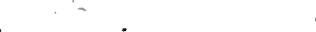


THIS BOOK IS DUE ON THE IAST DATE STAMPED BEIOW

AN INITIAL FINE OF 25 CENTS WILL BE ASSESSED FOR FAILURE TO RETURN THIS BOOK ON THE DATE DUE. THE PENALTY WILL INCREASE TO 50 CENTS ON THE FOURTH DAY AND TO \$1.00 ON THE SEVENTH DAY OVERDUE.

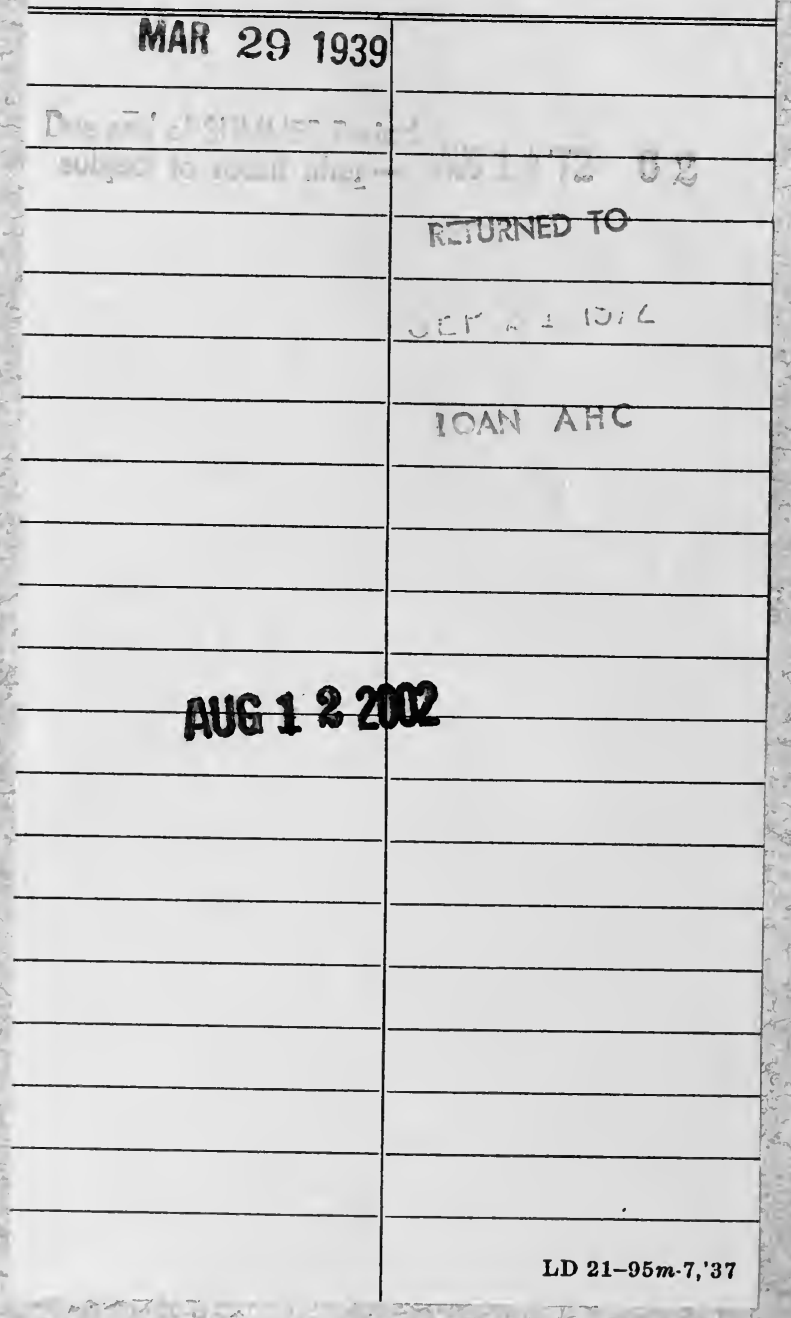




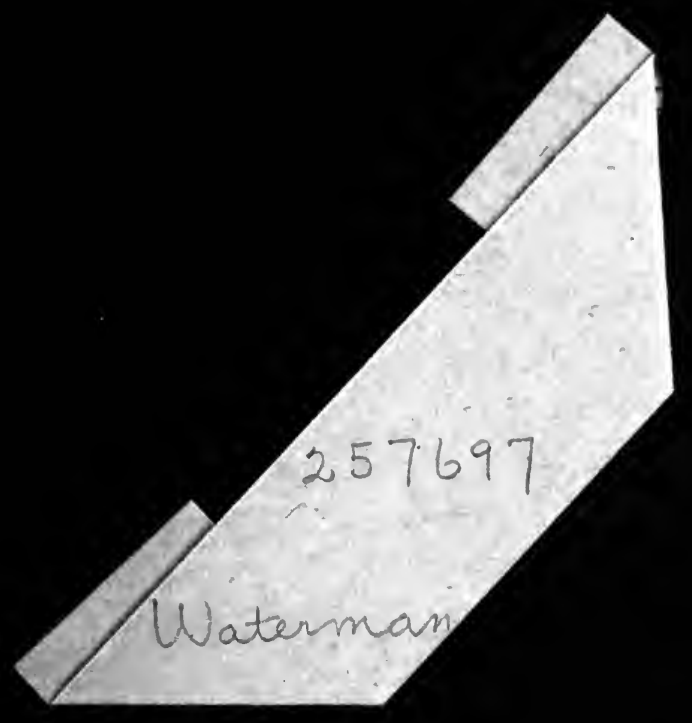

$y$ 
